\title{
Zhou Cong
}

\section{Teorema Ergódico Multiplicativo em Espaços Métricos de Curvatura \\ Não-positiva}

\section{DISSERTAÇÃO DE MESTRADO}

DEPARTAMENTO DE MATEMÁTICA

Programa de Pós-graduação em Matemática 
Zhou Cong

\section{Teorema Ergódico Multiplicativo em Espaços Métricos de Curvatura Não-positiva}

Dissertação apresentada ao Programa de Pós-graduação em Matemática da PUC-Rio como requisito parcial para obtenção do grau de Mestre em Matemática.

Orientador: Prof. Jairo da Silva Bochi 
Zhou Cong

\title{
Teorema Ergódico Multiplicativo em Espaços Métricos de Curvatura Não-positiva
}

\begin{abstract}
Dissertação apresentada ao Programa de Pós-graduação em Matemática da PUC-Rio como requisito parcial para obtenção do grau de Mestre em Matemática. Aprovada pela Comissão Examinadora abaixo assinada.
\end{abstract}

Prof. Jairo da Silva Bochi

Orientador

Pontifícia Universidade Católica do Rio de Janeiro

Prof. Nicolau Corção Saldanha

Pontifícia Universidade Católica do Rio de Janeiro

Prof. Rafael Oswaldo Ruggiero Rodriguez

Pontifícia Universidade Católica do Rio de Janeiro

Prof. Roberto Imbuzeiro Oliveira

Instituto Nacional de Matemática Pura e Aplicada

Prof. José Eugênio Leal

Coordenador de Pós-Graduação e Pesquisa do

Centro Técnico Científico

Pontifícia Universidade Católica do Rio de Janeiro 
Todos os direitos reservados. É proibida a reprodução total ou parcial do trabalho sem autorização da universidade, do autor e do orientador.

\section{Zhou Cong}

Graduou-se em Universidade Federal Fluminense em Julho de 2010 .

Ficha Catalográfica

Zhou Cong

Teorema Ergódico Multiplicativo em Espaços Métricos de Curvatura Não-positiva / Zhou Cong; orientador: Jairo da Silva Bochi. - Rio de Janeiro : PUC-Rio, Departamento de Matemática, 2013.

v., 70 f: il. ; $29,7 \mathrm{~cm}$

1. Dissertação (mestrado) - Pontifícia Universidade Católica do Rio de Janeiro, Departamento de Matemática.

Inclui referências bibliográficas.

1. Matemática - Dissertação. 2. Teoria Ergódica. 3. Isometria. 4. Curvatura não-positiva. 5. Teorema Ergódico Multiplicativo. I. Jairo da Silva Bochi. II. Pontifícia Universidade Católica do Rio de Janeiro. Departamento de Matemática. III. Título. 


\section{Agradecimentos}

Ao meu orientador e professor Jairo, quem dedicou intensamente em esclarecer as minhas dúvidas, pelas sugestões e correções neste dissertação.

Aos meus pais pelo apoio e dicas na linguagem TEX.

Ao professor Roberto Imbuzeiro, por apontar vários pontos que precisam ser esclarecidas.

Aos professores das instituições PUC, IMPA e UFF pelo ensinamento e apoio. Aos funcionários da PUC pelas soluções de problemas.

Aos colegas pela amizade e apoio.

À PUC-Rio pela bolsa de insensão e pela concretização da minha formação.

Ao CAPES e CNPq pelo apoio financeiro. 


\section{Resumo}

Zhou Cong; Jairo da Silva Bochi. Teorema Ergódico Multiplicativo em Espaços Métricos de Curvatura Não-positiva. Rio de Janeiro, 2013. 70p. Dissertação de Mestrado — Departamento de Matemática, Pontifícia Universidade Católica do Rio de Janeiro.

Apresentaremos uma versão de Teorema Ergódico Multiplicativo para cociclos subaditivos devido a Karlsson e Margulis. Como aplicação, analisaremos três exemplos de cociclos nos seguintes espaços: Grafo gerado por grupo livre em dois geradores, disco hiperbólico, espaço das matrizes positivas simétricas definidas. Também usaremos o Teorema de Karlsson e Margulis para mostrar o Teorema de Oseledets.

\section{Palavras-chave}

Teoria Ergódica; Isometria; Curvatura não-positiva; Teorema Ergódico Multiplicativo; 


\section{Abstract}

Zhou Cong; Jairo da Silva Bochi (Advisor). Multiplicative Ergodic Theorem in Nonpositively Curved Spaces. Rio de Janeiro, 2013. 70p. MSc. Dissertation — Departamento de Matemática, Pontifícia Universidade Católica do Rio de Janeiro.

We will show a version of Multiplicative Ergodic Theorem for subbaditive cocycles due to Karlsson and Margulis. As an application, we will analyze three examples of cocycles in following spaces: graph generated by free group of two generators, hyperbolic disc, space of positive definite symetric matrices. Also, we will use the Theorem of Karlsson and Margulis to prove Theorem of Oseledets.

\section{Keywords}

Ergodic Theory; Isometry; Non-positive curvature; Multiplicative Ergodic Theorem; 


\section{Sumário}

1 Introdução $\quad \mathbf{9}$

1.1 História e motivação 9

$\begin{array}{lll}1.2 & \text { Resumo dos capítulos } & 10\end{array}$

2 Enunciado do Teorema 12

2.1 Preliminares geométricos 12

2.2 Preliminares de Teoria Ergódica 16

2.3 Teorema de Karlsson e Margulis sobre cociclo de semicontrações 18

3 Primeiro Exemplo: Isometrias de uma Árvore $\quad 23$

3.1 Geometria em uma Árvore $\mathbb{F}_{2} \quad 23$

3.2 Cociclo em Grafo de Cayley 24

3.3 Aplicando Teorema de Kingman a $d\left(y_{0}, y_{n}\right) \quad 25$

3.4 Duas afirmações para mostrar que $A=\frac{1}{2} \quad 26$

3.5 Aplicação do Teorema $2.10 \quad 27$

4 Segundo Exemplo: Isometrias do Disco Hiperbólico 28

4.1 Definição do Cociclo de Isometrias 28

$\begin{array}{lll}4.2 & \text { Propriedades do Cociclo } u & 31\end{array}$

4.3 Aplicação do Teorema 2.10 34

4.4 Comportamento no caso $\omega=(\overline{1234}) \quad 35$

$5 \quad$ Teorema de Oseledets $\quad 37$

5.1 Teorema de Oseledets 37

$5.2 \operatorname{Pos}_{k}(\mathbb{R})$ como uma Variedade Riemanniana 38

5.3 Demonstração do Teorema de Oseledets 40

5.4 Exemplo de aplicação do Teorema de Oseledets 44

6 Preliminares Geométricos $\quad 48$

6.1 Um Lema Geométrico 48

6.2 Variedades de Curvatura Seccional Não-positiva 49

$7 \quad$ Propriedades de Cociclos Subaditivos $\quad \mathbf{5 2}$

7.1 Lema de Pliss Ergódico e algumas propriedades 52

7.2 Lema de Pliss Versão Fraca 54

7.3 Prova do Lema de Pliss Ergódico 58

7.4 Preparatórios para a demonstração do Teorema de Kingman 59

7.5 Prova do Teorema de Kingman 62

8 Prova do Teorema $\quad \mathbf{6 5}$

8.1 Escolhendo $\omega$ com boas propriedades 65

8.2 Construção da Sequência de Cauchy 66

8.3 Existência da geodésica $\gamma$ com as propriedades do teorema 67

$\begin{array}{ll}\text { Referências Bibliográficas } & 69\end{array}$ 


\section{Lista de Símbolos}

$(\Omega, \mathcal{A}, \mu)$ espaço de probabilidade

$(Y, d) \quad$ espaço métrico

$\mathbb{D} \quad$ disco unitário bidimensional, p.28

$\mathbb{F}_{2} \quad$ grupo livre em dois geradores

$\bar{b} \quad$ conjugado do número complexo $b$

$\overline{x y} \quad$ segmento geodésico de $x$ a $y$

A "drift" do cociclo, p.19

$a(\cdot, \cdot) \quad$ cociclo subaditivo, p.17

$G L_{k}(\mathbb{R})$ grupo das matrizes $k \times k$ reais invertíveis

Iso $(Y)$ grupo das isometrias do espaço métrico $Y$

$M^{*} \quad$ adjunta da matriz $M$

$\mathrm{Pos}_{k} \quad$ conjunto das matrizes matrizes $k \times k$ simétricas e positivas, p.38

Sym $_{k} \quad$ espaço das matrizes $k \times k$ simétricas, p.38

$u(\cdot, \cdot) \quad$ cociclo, p.16

$X \quad$ grafo de Cayley de $\mathbb{F}_{2}$, p.24

K-M essa sigla significa: Karlsson e Margulis. O termo "Teorema de K-M" refere-se ao Teorema de Karlsson e Margulis 2.10 da página 19 


\section{Introdução}

\subsection{História e motivação}

O surgimento dos Teoremas Ergódicos teve raiz no final do século XIX quando a Hipótese Ergódica começou a ser usada em termodinâmica, mecânica estatística devido a Boltzmann. Uma versão da hipótese ergódica pode ser formulada assim:

\section{"Para sistemas grandes de partículas interagindo em equilíbrio, a média temporal está perto da média espacial."}

Ela diz que o tempo que uma partícula encontra-se numa certa região no espaço das fases com a mesma energia é proporcional ao volume desta região quando o período é longo. Porém a formalização matemática da hipótese ergódica só começou a tomar forma em 1929, quando Bernard Osgood Koopman começou a investigar grupos de operadores unitários no espaço de Hilbert (sistemas Hamiltonianos). Os resultados de Koopman inspiraram John von Neumann a provar a primeira versão ([von Neumann, 1932]) do Teorema Ergódico (em $\left.L^{2}\right)$ :

Consideremos $(\Omega, \mathcal{A}, \mu)$ espaço de probabilidade, $T: \Omega \rightarrow \Omega$ preservando a probabilidade e $f: \Omega \rightarrow \mathbb{R}$ uma função.

Teorema 1.1 (von Neumann, 1931). Se T é transformação ergódica e $\int_{\Omega} f^{2} d \mu<\infty$, então

$$
\lim _{n \rightarrow \infty} \frac{1}{n} \sum_{k=0}^{n-1} f \circ T^{k} \omega=\int_{\Omega} f d \mu \text { em } L^{2} .
$$

Mais tarde, no mesmo ano, George David Birkhoff provou em [Birkhoff, 1931] uma versão de convergência q.t.p. do teorema: 
Teorema 1.2 (Birkhoff, 1931). Se $T$ é transformação ergódica e $\int_{\Omega} f^{+} d \mu<\infty$, então

$$
\lim _{n \rightarrow \infty} \frac{1}{n} \sum_{k=0}^{n-1} f \circ T^{k} \omega=\int_{\Omega} f d \mu, \text { para quase todo } \omega \in \Omega
$$

Estes são as duas versões clássicas do Teorema Ergódico. Em 1968, John Kingman generalizou o Teorema de Birkhoff ([Kingman, 1968]) para sequências de funções subaditivas.

Com o tempo, as demonstrações dos teoremas foram melhoradas e foram elaboradas outros variantes do teorema. Dentro das quais apareceram as versões multiplicativas do teorema, tratam de situações onde as "somas" de Birkhoff são substituídas por "produtos" não-comutativos: [Oseledets, 1968], [Kaimanovich, 1989], [Karlsson e Margulis, 1999], que são progressivamente mais gerais.

O resultado de $\mathrm{K} \mathrm{M}^{*}$ trata de propriedade de rastreamento sublinear para cociclos de semicontrações (inclusive as isometrias) de espaços métricos completo uniformemente convexo de curvatura não-positiva (em algum sentido que apresentaremos em diante). Já o Teorema de Oseledets descreve as taxas de crescimento de vetores sob produtos de matrizes. Foi Kaimanovich o pioneiro a mostrar como interpretar o Teorema de Oseledets de maneira geométrica, inspirando posteriormente resultados como o de K-M. Alguns resultados relacionados recentes são [Karlsson e Ledrappier, 2008], [Navas, 2011], [Tiozzo, 2012].

\subsection{Resumo dos capítulos}

Aqui focaremos no Teorema de [Karlsson e Margulis, 1999] e suas aplicações. Um dos objetivos principais desse texto é apresentar uma demonstração completa e detalhada desse resultado e do Teorema de Kingman. Também enunciaremos e mostraremos o Teorema de Oseledets. Obteremos o Teorema de Kingman como escólio da prova do Teorema de Karlsson e Margulis. Já o Teorema de Oseledets será basicamente obtido aplicando o Teorema de K-M como um caso particular.

O Capítulo 2 destina-se a definir e esclarecer os conceitos básicos que usaremos adiante. Além disso, enunciaremos o resultado principal. Nos dois capítulos seguintes analisaremos dois exemplos em detalhes. O primeiro

\footnotetext{
*Usaremos a sigla "K-M" para abreviar a expressão "Karlsson e Margulis" ao longo do texto.
} 
exemplo (Capítulo 3) é um cociclo na árvore gerado pelo grupo livre em dois geradores. O segundo exemplo (Capítulo 4) é um cociclo no disco com a métrica hiperbólica. Em seguida, no Capítulo 5, apresentaremos alguns conceitos elementares sobre o espaço das matrizes positivas e os utilizaremos para a demonstração do Teorema de Oseledets. Ainda no final do Capítulo 5, veremos mais um exemplo de cociclo de matrizes onde os expoentes de Lyapunov são distintos. Depois mostraremos em detalhes a Teorema de K-M no Capítulo 8. Os resultados geométricos a serem utilizados na demonstração estão no Capítulo 6. No Capítulo 7 mostraremos os resultados ergódicos a serem usados para a prova do Teorema K-M inclusive uma proposição profunda: uma versão generalizada do Lema de Pliss sobre os cociclos subaditivos, também mostrado por K-M, tendo outras aplicações interessantes.

Caso o leitor prefira seguir diretamente para a demonstração do Teorema de K-M, a ordem dos capítulos sugerida é $2 \rightarrow 6 \rightarrow 7 \rightarrow 8$. E uma vez que o Capítulo 2 esteja bem entendido, os Capítulos 3, 4 e 5 podem ser lidos da forma independente.

Durante a leitura de últimos dois capítulos, onde as demonstrações são relativamente técnicas, o leitor pode aproveitar da seguinte tabela que mostra a relação entre os conceitos principais apresentados ao longo do texto:

Lema de Pliss Ergódico 7.3 Lema Geométrico 6.1 Busemann NPC 2.5

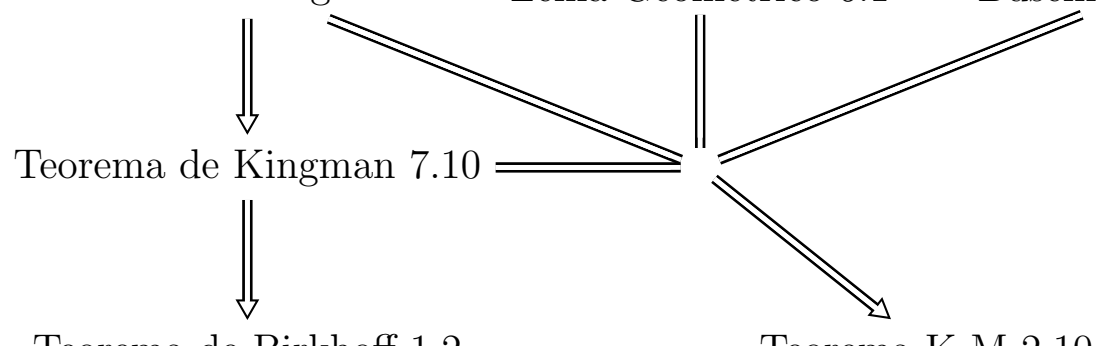

Teorema de Birkhoff 1.2

Teorema K-M 2.10 


\section{Enunciado do Teorema}

Este capítulo dedica-se à apresentação das definições geométricas e de teoria ergódica que serão usada ao longo do texto. Mostraremos algumas propriedades com respeito às definições e conceitos. No final do capítulo, enunciaremos o Teorema de Karlsson-Margulis 2.10.

\subsection{Preliminares geométricos}

Consideremos o espaço métrico $(Y, d)$. Sejam $x, y \in Y$, dizemos que $z \in Y$ é um ponto médio de $x$ e $y$ se:

$$
d(z, x)=d(z, y)=\frac{1}{2} d(x, y)
$$

Definição 2.1 (convexidade). Um espaço métrico $(Y, d)$ é dito convexo se quaisquer dois pontos possuem algum ponto médio.

O ponto médio não é único segundo a definição acima. Por exemplo, na esfera se escolhemos $x$ como polo norte e $y$ como polo sul, todo ponto no equador é ponto médio de $x$ e $y$.

Exemplo. $\left(\mathbb{Q}^{n}, d\right)$ conjunto dos números racionais com a métrica Euclidiana de $\mathbb{R}^{n}$ é um espaço métrico convexo (porém não é completo).

Definição 2.2 (geodésica). Uma aplicação contínua $\gamma: I \rightarrow Y$, onde $I$ é um intervalo de $\mathbb{R}$, é dita geodésica. Se existe constante $v \in(0, \infty)$ tal que:

$$
d(\gamma(s), \gamma(t))=v|s-t|
$$

para quaisquer $s, t \in I$. Chamamos $v$ de velocidade da geodésica. Quando $v=1$ dizemos que a geodésica é de velocidade unitária.

Se o espaço métrico $(Y, d)$ é convexo e completo, então quaisquer dois pontos podem ser conectados por algum segmento geodésico (podendo não ser único).

A seguinte definição é devida a Clarkson 1936 (no contexto de espaços de Banach) 
Definição 2.3 (convexidade uniforme). Um espaço convexo $(Y, d)$ é dito uniformemente convexo se existe uma função contínua decrescente $g$ : $[0,1] \rightarrow[0,1], \operatorname{com} g(0)=1, g(1)=0$ e tal que:

$$
\frac{d\left(z, m_{x y}\right)}{R} \leq g\left(\frac{d(x, y)}{2 R}\right), \quad \forall x, y, z \in Y,
$$

onde $m_{x y}$ é um ponto médio de $x$ e $y$, e $R:=\max \{d(x, z), d(y, z)\}$.

Essa definição é equivalente a: dado $\epsilon>0$ existe $\delta(\epsilon)>0$ contínua e crescente tal que para todo $x, y, z \in Y$, e $m_{x y}$ ponto médio de $x$ e $y$, vale:

$$
d\left(z, m_{x y}\right) \leq R(1-\epsilon), \quad \forall x, y, z \operatorname{com} d(x, y) \leq R \delta .
$$

À primeira vista, aparentemente o número "2" no quociente da equação (2.1) é supérfluo, mas existem motivos para essa notação, mostraremos isso abaixo:

$O$ espaço Euclidiano $\mathbb{R}^{n}$ é uniformemente convexo. De fato dado um triângulo com vértices $x, y, z$, suponhamos sem perda de generalidade $R=$ $d(z, x)$ e seja $\theta=\angle z m_{x y} x$. Daí $\theta \geq \frac{\pi}{2}$ e pela Lei de Cosseno:

$$
\begin{aligned}
& \left(d\left(z, m_{x y}\right)\right)^{2}=(d(z, x))^{2}-\left(\frac{d(x, y)}{2}\right)^{2}+2(\cos \theta) d\left(m_{x y}, z\right) d\left(m_{x y}, x\right) \\
\Longrightarrow & \left(\frac{d\left(z, m_{x y}\right)}{R}\right)^{2}=1-\left(\frac{d(x, y)}{2 R}\right)^{2}+2 \cos \theta\left(\frac{d(x, y)}{2 R}\right) \\
\Longrightarrow & \frac{d\left(z, m_{x y}\right)}{R} \leq\left(1-\left(\frac{d(x, y)}{2 R}\right)^{\frac{1}{2}}\right)^{2},
\end{aligned}
$$

onde na última desigualdade usamos $\cos \theta \leq 0$. Definimos $g(t):=\left(1-t^{2}\right)^{\frac{1}{2}}$, a desigualdade acima implica:

$$
\frac{d\left(z, m_{x y}\right)}{R} \leq g\left(\frac{d(x, y)}{2 R}\right)
$$

A função $g$ é uma função contínua, decrescente com $g(0)=1, g(1)=0$ e não depende da escolha dos pontos. Isto mostra que $\mathbb{R}^{n}$ é uniformemente convexo.

Observação. Para uma noção intuitiva da convexidade uniforme, comparamos os três triângulos do $\mathbb{R}^{2}$ na Figura 2.1. Note que $d\left(z, m_{x y}\right)$ é sempre menor que $R$, e a medida que a distância entre vértices $x$ e $y$ diminui, $d\left(z, m_{x y}\right)$ aproxima de $R$ por baixo. Essa é a caraterização da propriedade de convexidade uniforme. 

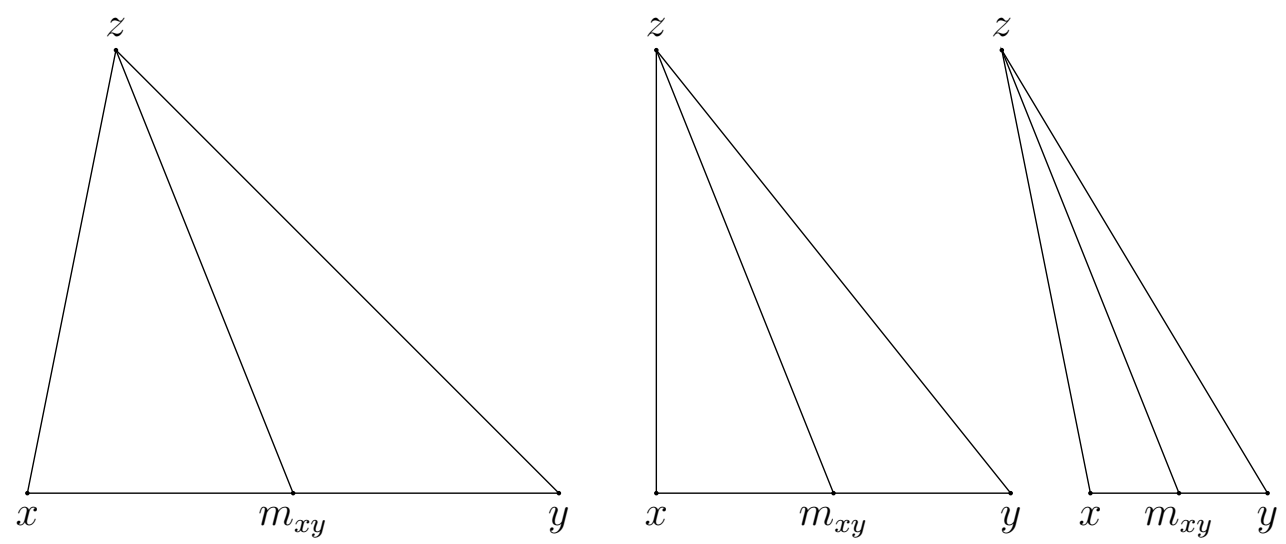

Figura 2.1: Observe que $\overline{z m}_{x y}$ é sempre menor que o lado maior $\overline{z y}$.

Posteriormente, na Proposição 6.2, deduziremos que as variedades Riemannianas de curvatura não-positiva, completas e simplesmente conexas são uniformemente convexas. A mesma função $g(t)=\left(1-t^{2}\right)^{\frac{1}{2}}$ escolhida no caso Euclidiano pode ser usada para estes espaços (e mais geralmente para os espaços $\operatorname{CAT}(0))$.

Em contraste com os exemplos acima, uma variedade que não é uniformemente convexa é a esfera. De fato, sejam $z$ o polo norte, $x$ e $y$ dois pontos distintos no equador, logo podemos tomar um ponto médio $m_{x y}$ no equador, daí $d\left(z, m_{x y}\right)=R$ (isto mostra que a condição de curvatura não-positiva é necessária para garantir a convexidade uniforme). Um outro exemplo de variedade não é uniformemente convexo é o cilindro $\left\{(x, y, z) \in \mathbb{R}^{3} \mid x^{2}+y^{2}=1\right\}$, escolhendo $x=(1,0,0), y=(-1,0,0), z=(0,-1,0)$, temos $m_{x y}=(0,1,0)$, daí: $d\left(z, m_{x y}\right)=\pi>\frac{\pi}{2}=R$ (a condição do espaço ser simplesmente conexo também é necessária).

Proposição 2.4. Se $(Y, d)$ é um espaço métrico uniformemente convexo. Então dados quaisquer dois pontos do $Y$, existe um único ponto médio entre eles.

Prova. Suponhamos, por absurdo, que existem os pontos $x, y, z, w \in Y$ tais que $z$ e $w$ são pontos médios entre $x$ e $y$. Sejam $R:=\frac{d(x, y)}{2}$ e $m_{z w}$ um ponto médio de $z$ e $w$. Pela convexidade uniforme temos:

$$
\frac{d\left(x, m_{z w}\right)}{R} \leq g\left(\frac{d(z, w)}{2 R}\right)<1
$$

daí $d\left(x, m_{z w}\right)<R$. Analogamente, $d\left(y, m_{z w}\right)<R$. Portanto temos:

$$
d(x, y) \leq d\left(x, m_{z w}\right)+d\left(y, m_{z w}\right)<2 R
$$

contradizendo a hipótese de $2 R=d(x, y)$. 


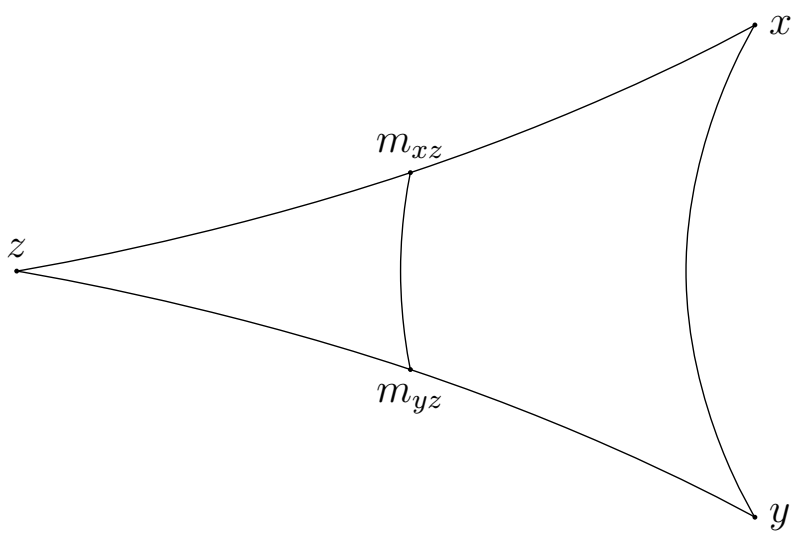

Figura 2.2: Esboço do triângulo no espaço Hiperbólico.

Como consequência da proposição, dados dois pontos distintos de um espaço uniformemente convexo, o segmento geodésico ligando estes pontos é único. Mas isso não garante que o prolongamento do segmento geodésico é único, por exemplo, na árvore como no Capítulo 3.

Definição 2.5 (curvatura não-positiva). Dizemos que um espaço métrico convexo $(Y, d)$ tem curvatura não-positiva (no sentido de Busemann) ${ }^{a}$ se para quaisquer $x, y, z \in Y$, e quaisquer pontos médios $m_{x z}$ de $\overline{x z}$ e $m_{y z}$ de $\overline{y z}$ satisfazem:

$$
d\left(m_{x z}, m_{y z}\right) \leq \frac{1}{2} d(x, y),
$$

como mostra no esboço da Figura 2.2.

${ }^{a}$ Em inglês: Busemann NPC. A sigla NPC significa Nonpositive Curvature.

A condição acima (2.3) equivale à propriedade que para quaisquer duas geodésicas $\gamma_{1}, \gamma_{2}:[0,1] \rightarrow Y$ começando no ponto $z$ temos (cf. [Jost, p.46]):

$$
t \longmapsto \frac{1}{t} d\left(\gamma_{1}(t), \gamma_{2}(t)\right) \quad \text { é não-decrescente. }
$$

Em particular, as variedades Riemannianas completas de curvaturas seccionais não-positivas satisfazem a condição de curvatura não-positiva de Busemann; Provaremos isso na Proposição 6.2.

Observação 1. Os espaços CAT(0) (seguindo a definição de [M. R. Bridson e A. Haefliger, p.158-159]) são um tipo particular de espaços com curvatura não-positiva de Busemann. Convém lembrar que a comparação da definição de Busemann é feita somente para o segmento ligando os pontos médios, enquanto na definição $\mathrm{CAT}(0)$ a comparação é feita num segmento arbitrário ligando as arestas triângulo. Isso faz com que o conceito de Busemann seja mais geral que os espaços CAT(0) (para um exemplo de espaço de Busemann que não é $\operatorname{CAT}(0)$ cf. [Jost, p.55]). 
Observação 2. Alguns autores como [Jost] chamam o conceito da Definição 2.5 de curvatura não-positiva global no sentido de Busemann. Existe um outro conceito local de curvatura não-positiva de Busemann que não usaremos aqui. Este exige que para cada ponto $y \in Y$, exista uma vizinhança $V_{y}$ de $y$ satisfazendo a condição de curvatura não-positiva no sentido de Busemann. Este conceito é, em geral, mais fraco que a condição da Definição 2.5.

\subsection{Preliminares de Teoria Ergódica}

Ao longo do texto denotaremos $(\Omega, \mathcal{A}, \mu)$ um espaço de probabilidade e $(G, *)$ um monoide* . Consideremos $T: \Omega \rightarrow \Omega$ uma transformação mensurável preservando a probabilidade.

Lembramos que uma transformação $T: \Omega \rightarrow \Omega$ é dita preserva a probabilidade se $T$ é mensurável e $\mu\left(T^{-1}(C)\right)=\mu(C)$ para todo $C$ mensurável. Além disso, dizemos que $T$ é ergódica se $T$ preserva a probabilidade e para todo conjunto mensurável $C \subset \Omega$ tal que $T^{-1}(C)=C \bmod \mu$ temos $\mu(C) \in\{0,1\}$. Para mais informações sobre esses conceitos ver [Mañé].

Definição 2.6 (cociclo). Dizemos que a aplicação $u: \mathbb{N} \times \Omega \rightarrow G$ é cociclo (com respeito à transformação T) quando u satisfaz as propriedades:

1. $u(0, \omega)=e \quad$ onde e é o elemento neutro de $G$,

2. $u(n+m, \omega)=u(n, \omega) * u\left(m, T^{n} \omega\right) \quad \forall n, m \in \mathbb{N}^{*}$.

O Item 2 dessa definição equivale ao:

$$
u(n, \omega)=f(\omega) * f(T \omega) * \cdots * f\left(T^{n-1} \omega\right) \quad \forall n \in \mathbb{N}, \forall \omega \in \Omega,
$$

onde $f(\omega):=u(1, \omega)$.

Pela ordem em que os termos são multiplicados, também dizemos que $u$ é um cociclo à direita. No Capítulo 5 definiremos cociclo à esquerda, onde os termos são multiplicados na ordem contrária. Essa distinção é importante pois os resultados que apresentaremos adiante só valem para os cociclos à direita.

Recordamos que uma sequência de números reais $\left\{a_{n}\right\}_{n \in \mathbb{N}}$ é chamada subaditiva se:

$$
a_{n+m} \leq a_{n}+a_{m} \quad \forall n, m \in \mathbb{N}
$$

Generalizaremos isto para cociclos reais:

${ }^{*}$ Dizemos que $(G, *)$ é monoide quando para todos $g, h \in G, g * h \in G$ e existe um elemento neutro $e \in G$ (mas $g^{-1}$ pode não existir). 
Definição 2.7 (cociclo subaditivo). Uma função $a: \mathbb{N} \times \Omega \rightarrow \mathbb{R}$ definida sobre os números reais é dita cociclo subaditivo quando satisfaz:

$$
a(n+m, \omega) \leq a\left(n, T^{m} \omega\right)+a(m, \omega) \quad \forall n, m \in \mathbb{N} \quad \forall \omega \in \Omega .
$$

Quando a desigualdade acima é uma igualdade, dizemos que a é um cociclo aditivo.

É importante ressaltar que, em geral, um cociclo subaditivo não é cociclo, apesar do que o seu nome sugere. A seguir provaremos um resultado bem conhecido das sequências subaditivas:

Proposição 2.8. Seja $\left\{a_{n}\right\}_{n \in \mathbb{N}}$ uma sequência subaditiva. Então:

$$
\lim _{n \rightarrow \infty} \frac{a_{n}}{n} \quad \text { existe e assume valor no conjunto }\left[-\infty, a_{1}\right] .
$$

Prova. Definimos: $A:=\inf _{N \geq 1} \frac{a_{N}}{N}$. Vamos mostrar que $A \geq \limsup _{n \rightarrow \infty} \frac{a_{n}}{n}$. Pela subaditividade temos $a_{n} \leq n a_{1}<\infty$, tomando supremo:

$$
\limsup _{n \rightarrow \infty} \frac{a_{n}}{n} \leq a_{1}<\infty
$$

Dado $N \geq 1$, para todo $n \in \mathbb{N}$ podemos escrever:

$$
\begin{aligned}
n & =k_{n} N+r_{n} \quad r_{n}, k_{n} \in \mathbb{Z} \text { e } 1 \leq r_{n} \leq N \\
\Longrightarrow & \frac{1}{N}=\frac{k_{n}}{n}+\frac{r_{n}}{n N} .
\end{aligned}
$$

Fazendo $n \rightarrow \infty$ em ambos lados, temos:

$$
\lim _{n \rightarrow \infty} \frac{k_{n}}{n}=\frac{1}{N}
$$

Por outro lado, pela subaditividade,

$$
\frac{a_{n}}{n} \leq \frac{k_{n} a_{N}+a_{r_{n}}}{n}
$$

Observe que $\lim _{n \rightarrow \infty} \frac{a_{r_{n}}}{n}=0$. Tomemos uma subsequência $n_{i} \rightarrow \infty$ tal que $\lim _{n \rightarrow \infty} \frac{a_{n_{i}}}{n_{i}}=\limsup _{n \rightarrow \infty} \frac{a_{n}}{n}$. Então a desigualdade acima junto com a equação (2.6) implicam:

$$
\limsup _{n \rightarrow \infty} \frac{a_{n}}{n} \leq \frac{a_{N}}{N}
$$


Como $N \geq 1$ é arbitrário, concluímos que $\limsup _{n \rightarrow \infty} \frac{a_{n}}{n} \leq A$.

Se $a$ é cociclo subaditivo então as integrais $a_{n}:=\int_{\Omega} a(n, \omega) d \mu$, se existirem, formam sequência subaditiva. Pela Proposição 2.8, o limite $\lim _{n \rightarrow \infty} \frac{a_{n}}{n}$ existe. Ao longo do texto, vamos usar a notação:

$$
A:=\lim _{n \rightarrow \infty} \frac{1}{n} \int_{\Omega} a(n, \omega) d \mu \text {. }
$$

Por motivos que veremos mais adiante, chamaremos $A$ de "drift". A seguir, enunciaremos o Teorema Ergódico de Kingman:

Teorema 2.9 (Kingman). Supondo que $T$ é ergódico, a um cociclo subaditivo e $A>-\infty$. Então:

$$
\text { existe } \lim _{n \rightarrow \infty} \frac{1}{n} a(n, \omega)=A \quad \text { para quase todo } \omega \in \Omega \text {. }
$$

Usaremos este teorema nos Capítulos 3 e 8, para mostrar o Teorema K-M. A prova do Teorema de Kingman pode ser encontrada na Seção 7.5.

\subsection{Teorema de Karlsson e Margulis sobre cociclo de semicontrações}

Dizemos que uma transformação $\varphi: Y \rightarrow Y$ de espaço métrico $(Y, d)$ é uma semicontração quando:

$$
d(\varphi(x), \varphi(y)) \leq d(x, y), \quad \text { para todos } x, y \in Y .
$$

Caso a desigualdade acima é uma igualdade, dizemos que $\varphi$ é uma isometria. Quando $G$ é um monoide de semicontrações, chamamos um cociclo $u$ : $\mathbb{N} \times \Omega \rightarrow G$ de cociclo de semicontrações (do mesmo modo, definimos cociclo de isometrias).

Dado conjunto $G$ das transformações contínuas de um espaço topológico $Y^{\dagger}$ definimos a topologia compacto-aberto: Nessa topologia a base de vizinhanças de um elemento $f \in G$ é formado por conjuntos do tipo:

$$
\bigcap_{i=1}^{n}\left\{g \in G \mid g\left(K_{i}\right) \subset A_{i}\right\},
$$

onde $K_{i}$ 's são subconjuntos compactos de $Y$ e $A_{i}$ 's são subconjuntos abertos

\footnotetext{
${ }^{\dagger}$ Em particular podemos considerar $G$ um monoide de semicontrações e $Y$ um espaço métrico com a topologia induzida pela métrica.
} 
de $Y$. Além disso:

$$
f\left(K_{i}\right) \subset A_{i}, \quad \text { para todo } i \in\{1, \ldots, n\}
$$

Dizemos que um cociclo de semicontrações $u$ é mensurável quando $f(\omega):=u(1, \omega)$ é mensurável. Isso junto com a equação (2.5) garantem que $u(n, \omega)$ é mensurável para todo $n \in \mathbb{N}$. Portanto, exigir a mensurabilidade de $u(1, \omega)$ já basta.

Com os conceitos acima, enunciamos o Teorema K-M:

Teorema 2.10 (Karlsson-Margulis). Consideremos $(Y, d)$ um espaço métrico completo uniformemente convexo de curvatura não-positiva (no sentido de Busemann). Seja $u: \mathbb{N} \times \Omega \rightarrow G$ um cociclo de semicontrações ${ }^{a}$ mensurável em $Y$. Fixe $y \in Y$, denotamos $y_{n}(\omega):=u(n, \omega) y$. Assumimos também que u tem primeiro momento finito, isto é:

$$
\int_{\Omega} d\left(y, y_{1}(\omega)\right) d \mu<\infty
$$

Então temos as seguintes propriedades:

1. $\exists A \geq 0$ tal que $\lim _{n \rightarrow \infty} \frac{d\left(y, y_{n}(\omega)\right)}{n}=$ A para quase todo $\omega \in \Omega$.

2. Se $A>0$, então para quase todo $\omega \in \Omega$ existe uma geodésica $\gamma(\cdot, \omega)$ de velocidade unitária em $Y$ de modo que $\gamma(0, \omega)=y$ e

$$
\lim _{n \rightarrow \infty} \frac{d\left(\gamma(n A, \omega), y_{n}(\omega)\right)}{n}=0
$$

${ }^{a}$ Em particular, o teorema vale para cociclos de isometrias.

Definição 2.11 (drift). Sejam u um cociclo de semicontrações em $Y$, $y \in Y$ como no Teorema anterior 2.10. Seja $A \geq 0$ com as mesmas propriedades do teorema. Chamamos $A$ de drift do cociclo $u$ ou simplesmente de drift quando não há ambiguidades. Para cada $\omega \in \Omega$, usaremos uma convenção: definimos $A(\omega)$ como o limite (se existir):

$$
A(\omega):=\lim _{n \rightarrow \infty} \frac{d\left(y, y_{n}(\omega)\right)}{n} .
$$

Dizemos que $A(\omega)$ é o drift de $\omega$.

Exemplo. Observa-se que o Teorema de Birkhoff é um caso particular do Teorema 2.10 para $Y:=\mathbb{R}$ e $y:=0$. Seja $f$ a função como no Teorema 
de Birkhoff 1.2, colocamos $u(1, \omega) x:=x+f(\omega)$. Nesse caso a condição de integrabilidade fica:

$$
\int_{\Omega} d(y, y+f(\omega)) d \mu=\int_{\Omega} f^{+}(\omega) d \mu<\infty
$$

Quando $\left|\int_{\Omega} f d \mu\right|>0$ a conclusão do Teorema de Birkhoff é:

$$
\lim _{n \rightarrow \infty} \frac{1}{n}\left|y_{n}-\int_{\Omega} f d \mu\right|=0 \quad \text { para quase todo } \omega \in \Omega
$$

A equação acima na linguagem do Teorema K-M significa que:

$$
\lim _{n \rightarrow \infty} \frac{d\left(\gamma(n A), y_{n}\right)}{n}=0 \quad \text { para quase todo } \omega \in \Omega
$$

onde $A=\left|\int_{\Omega} f d \mu\right|>0$ e $\gamma(t)=t$ se o integral $\int_{\Omega} f d \mu$ é positivo. Caso contrário, temos $\gamma(t)=-t$.

Como vimos no exemplo acima, no caso do Teorema de Birkhoff, a geodésica $\gamma$ é "deterministica", isto é, não depende de $\omega$. Mas em geral a geodésica do Teorema K-M depende de $\omega$, veremos isso nos Capítulos 3 e 4. A razão das geodésicas não serem determinísticas em geral está contida na seguinte corolário:

Corolário 2.12. Sob as mesmas hipóteses e notações do Teorema 2.10, suponhamos que $A>0$ e $\omega \in \Omega$ é tal que existem as geodésicas $\gamma(\cdot, \omega)$ e $\gamma(\cdot, T \omega)$ de velocidades unitárias tais que:

$$
\lim _{n \rightarrow \infty} \frac{d\left(\gamma(n A, \omega), y_{n}(\omega)\right)}{n}=\lim _{n \rightarrow \infty} \frac{d\left(\gamma(n A, \omega), y_{n}(T \omega)\right)}{n}=0 .
$$

Então:

$$
\lim _{n \rightarrow \infty} \frac{d(\gamma(n A, \omega), u(1, \omega) \gamma(n A, T \omega))}{n}=0 .
$$

Em outras palavras, $\gamma(\cdot, \omega)$ e $\gamma(\cdot, T \omega)$ têm o mesmo "ponto no infinito".

Como podemos ver acima, as geodésicas só coincidirão se $u(1, \omega)$ preserva essas geodésicas. No caso do Teorema de Birkhoff, $u(1, \omega)$ são as translações, portanto preservam as geodésicas de $\mathbb{R}$. Mas em espaços de dimensões maiores isso não pode não acontecer, veremos nos capítulos seguintes.

Agora mostremos mais duas propriedades básicas relacionados ao Teorema K-M:

Proposição 2.13. Sob as mesmas notações do Teorema 2.10, a condição de integrabilidade (2.7) e o valor de drift A não dependem da escolha do 
ponto y no espaço $Y$.

Prova. Fixamos um outro $\tilde{y} \in \Omega$ e seja $\tilde{y}_{n}(\omega):=u(n, \omega) \tilde{y}$. Pela desigualdade triangular:

$$
\begin{aligned}
d\left(\tilde{y}, \tilde{y}_{1}(\omega)\right) & \leq d(\tilde{y}, y)+d\left(y, y_{1}(\omega)\right)+d\left(y_{1}(\omega), \tilde{y}_{1}(\omega)\right) \\
& \leq 2 d(\tilde{y}, y)+d\left(y, y_{1}(\omega)\right) .
\end{aligned}
$$

Integrando ambos lados em $\Omega$ :

$$
\int_{\Omega} d\left(\tilde{y}, \tilde{y}_{1}(\omega)\right) d \mu \leq 2 d(\tilde{y}, y)+\int_{\Omega} d\left(y, y_{1}(\omega)\right) d \mu \leq \infty
$$

Isto mostra que a condição de integrabilidade não depende de $y$.

Agora vamos mostrar o mesmo para o drift $A$. Novamente usando desigualdade triangular:

$$
\begin{aligned}
d\left(\tilde{y}, \tilde{y}_{n}(\omega)\right) & \leq d(\tilde{y}, y)+d\left(y, y_{n}(\omega)\right)+d\left(y_{n}(\omega), \tilde{y}_{n}(\omega)\right) \\
& \leq 2 d(\tilde{y}, y)+d\left(y, y_{n}(\omega)\right) .
\end{aligned}
$$

Dividindo por $n$ e levando $n \rightarrow \infty$ temos:

$$
\lim _{n \rightarrow \infty} \frac{d\left(\tilde{y}, \tilde{y}_{n}(\omega)\right)}{n} \leq \lim _{n \rightarrow \infty} \frac{2 d(\tilde{y}, y)+d\left(y, y_{n}(\omega)\right)}{n}=\lim _{n \rightarrow \infty} \frac{d\left(y, y_{n}(\omega)\right)}{n}
$$

Além disso, observamos que o Item 1 do Teorema 2.10 é uma consequência do Teorema de Kingman.

Afirmação 2.14. Para quase todo $\omega \in \Omega$, o limite

$$
\lim _{n \rightarrow \infty} \frac{1}{n} d\left(y, y_{n}(\omega)\right)=A \quad \text { para quase todo } \omega \in \Omega
$$

Prova. Definimos $a(n, \omega):=d\left(y, y_{n}\right)$, pela desigualdade triangular e $u$ ser cociclo de semi-contrações:

$$
\begin{aligned}
a(n+m, \omega)=d\left(y, y_{n+m}\right) & \leq d\left(y, y_{m}\right)+d\left(y_{m}, y_{n+m}\right) \\
& =d\left(y, y_{m}\right)+d\left(u(m, \omega) y, u(m, \omega) \circ u\left(n, T^{m} \omega\right) y\right) \\
& \leq a(m, \omega)+d\left(y, u\left(n, T^{m} \omega\right) y\right) \\
& =a(m, \omega)+a\left(n, T^{m} \omega\right),
\end{aligned}
$$


portanto $a$ é um cociclo subaditivo. Notemos que por hipótese de momento finito (2.7):

$$
\int_{\Omega} a^{+}(1, \omega) d \mu=\int_{\Omega} d\left(y, y_{1}(\omega)\right) d \mu<\infty
$$

$a_{n}:=\int_{\Omega} a(n, \omega) d \mu$ é finito e formam uma sequência subaditiva. Aplicando o Teorema de Kingman 2.9 temos:

$$
\lim _{n \rightarrow \infty} \frac{1}{n} d\left(y, y_{n}(\omega)\right)=A \geq 0 \quad \text { para quase todo } \omega \in \Omega .
$$

Observação. Como consequência da demonstração e da Proposição 2.13, convém destacar a seguinte relação para o drift:

$$
A=\lim _{n \rightarrow \infty} \frac{1}{n} \int_{\Omega} d\left(y, y_{n}(\omega)\right) d \mu=\lim _{n \rightarrow \infty} \frac{1}{n} d\left(y, y_{n}(\omega)\right),
$$

para quase todo $\omega \in \Omega$ e para todo $y \in Y$. 


\section{Primeiro Exemplo: Isometrias de uma Árvore}

Veremos que uma árvore simétrica com 4 arestas incidindo em cada vértice satisfaz as condições geométricas necessárias para a aplicação do Teorema de K-M 2.10. Definimos um cociclo sobre o grupo de isometrias dessa árvore e mostraremos que o seu drift $A$ é positivo.

\subsection{Geometria em uma Árvore $\mathbb{F}_{2}$}

Um grafo $X$ é união de arestas com identificação nos extremos, que são chamado de vértices. O grau de um vértice é o número de tais identificações (para definições mais precisas, ver [Bollobás]). Seja $X$ um grafo conexo tal que cada vértice tem grau finito. Para cada aresta, fixe uma parametrização tomando valores no intervalo $[0,1]$. Usando estas parametrizações, podemos definir comprimento de um caminho no grafo. Definimos a distância $d$ entre dois pontos do grafo como o menor comprimento de um caminho ligando os pontos. Então o espaço métrico resultante $(X, d)$ é convexo e completo.

Definição 3.1 (árvore). Dizemos que um grafo $X$ é uma árvore quando é conexo e não possui ciclos ou, equivalentemente, é simplesmente conexo.

Proposição 3.2. Seja $X$ uma árvore. Então $(X, d)$ é um espaço métrico uniformemente convexo de curvatura não-positiva.

Prova da Convexidade Uniforme. Sejam $x, y, z \in X$ três pontos distintos, como $X$ é simplesmente convexo, existe um único ponto médio $m_{x y}$ de $x$ e $y$. Notemos $m_{x y}$ está na geodésica de $x$ a $y$, pela propriedade mencionado anteriormente temos $m_{x y} \in \overline{z x} \cup \overline{z y}$. Suponhamos sem perda de generalidade que $m_{x y} \in \overline{z x}$, definimos $R:=\max (d(z, x), d(z, y))=d(z, x)$. Daí:

$$
\begin{aligned}
& d\left(z, m_{x y}\right)+d\left(m_{x y}, x\right)=d(z, x) \\
\Longrightarrow & d\left(z, m_{x y}\right)+\frac{d(x, y)}{2}=R \\
\Longrightarrow & \frac{d\left(z, m_{x y}\right)}{R}=1-\frac{d(x, y)}{2 R}=g\left(\frac{d(x, y)}{2 R}\right),
\end{aligned}
$$


onde $g(x):=1-x$ é contínua, decrescente com $g(0)=1$. Portanto $X$ é uniformemente convexo.

Prova da Curvatura não-positiva. Vamos provar que a condição (2.4) é satisfeita. Seja $z \in X$ e sejam $\gamma_{1}, \gamma_{2}:[0,1] \rightarrow X$ geodésicas partindo de ponto $z$ de velocidades $v_{1}$ e $v_{2}$, respectivamente. Suponhamos, sem perda de generalidade, que $\gamma_{1} \neq \gamma_{2}$ e que $v_{1} \geq v_{2}$. Definimos também $t_{0}$ como o tempo total que a geodésica $\gamma_{2}$ "se mantém na trajetória" de $\gamma_{1}$, isto é:

$$
t_{0}=\sup \left\{t \in[0,1] \mid \gamma_{2}(t)=\gamma_{1}\left(\frac{v_{2} t}{v_{1}}\right)\right\} .
$$

Logo temos:

$$
d\left(\gamma_{1}(t), \gamma_{2}(t)\right)= \begin{cases}\left(v_{1}-v_{2}\right) t & \text { se } 0 \leq t \leq t_{0} \\ \left(v_{1}+v_{2}\right) t-2 v_{2} t_{0} & \text { se } t>t_{0}\end{cases}
$$

Em particular, vale a condição (2.4).

\subsection{Cociclo em Grafo de Cayley}

Seja $\mathbb{F}_{2}$ o grupo livre em dois geradores $a$ e $b$. Daqui em diante consideremos $X$ o grafo de Cayley associado a este grupo e este conjunto de geradores (cf. Fig. 3.1). Ou seja, $X$ é o grafo cujo os vértices são identificados como elementos do grupo $\mathbb{F}_{2}$, e existe uma aresta conectando dois elementos $g_{1}, g_{2} \in \mathbb{F}_{2}$ se e somente se:

$$
g_{1}=g_{2} * h \quad \text { onde } h \in\left\{a, b, a^{-1}, b^{-1}\right\}
$$

Definimos $\Omega$ sendo o conjunto $\left\{a, b, a^{-1}, b^{-1}\right\}^{\mathbb{N} *}$ e usamos a notação $\omega=\left\{\omega_{i}\right\}_{i \in \mathbb{N}}$ para denotar os elementos de $\Omega$. Seja $\mu$ a medida de Bernoulli com pesos todos iguais a $1 / 4$ e $T: \Omega \rightarrow \Omega$ o shift para esquerda ${ }^{\dagger}$. Como é conhecido, a medida $\mu$ é $T$-invariante e ergódica.

Seja $G$ o grupo de isometrias de $X$ gerados por multiplicação à esquerda pelos elementos $a$ e $b$. Usamos e para denotar o elemento neutro de $G$. Seja $u: \mathbb{N} \times \Omega \longrightarrow G$ o cociclo definido por:

$$
u(n, \omega)= \begin{cases}\omega_{0} * \omega_{1} * \ldots * \omega_{n-1} & \text { se } n \geq 1, \\ e & \text { se } n=0 .\end{cases}
$$

\footnotetext{
${ }^{*} \mathrm{O}$ espaço das sequências unilaterais nos símbolos $a, b, a^{-1}, b^{-1}$

†Isto é, $T\left(\left\{\omega_{i}\right\}\right)=\left\{\omega_{i+1}\right\}$
} 


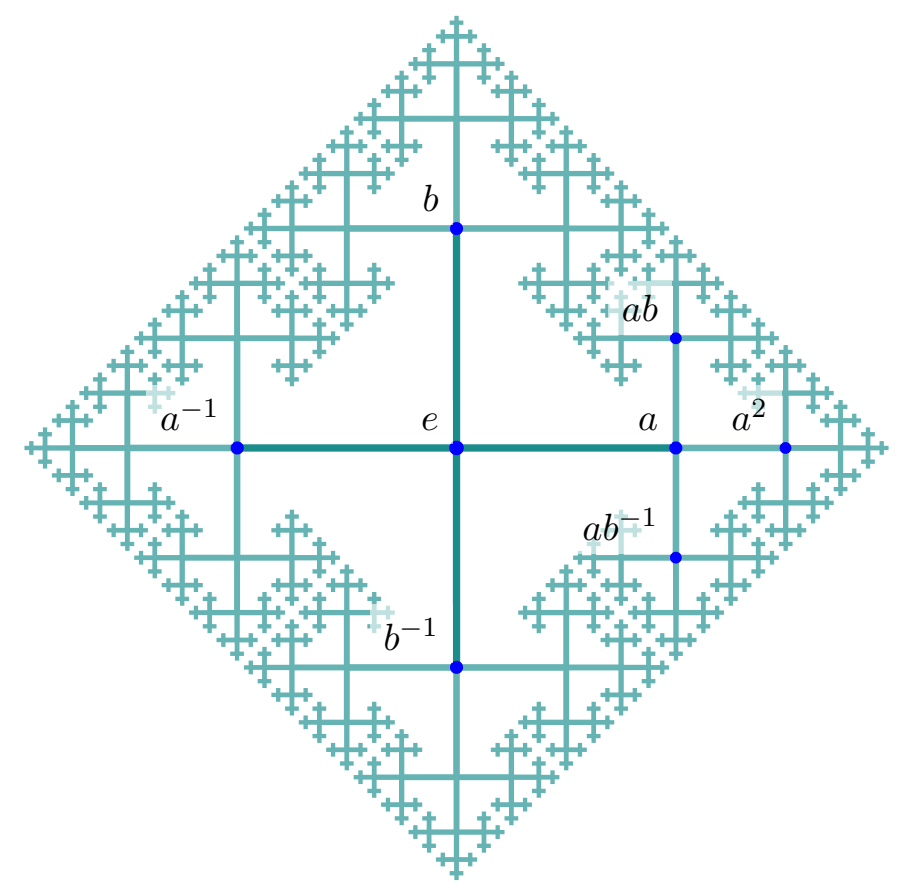

Figura 3.1: Um esboço do grafo de Cayley como fractal.

Dado qualquer vértice $y_{0}$ no grafo, e definindo $y_{i}=u(i, \omega) y_{0}$. Segue imediatamente que $y_{i}$ e $y_{i+1}$ são vértices adjacentes, para quaisquer $i \in \mathbb{N}$. Dizemos que a sequência $\left\{y_{i}\right\}_{i \in \mathbb{N}}$ é um passeio aleatório simples. Mostraremos, nas próximas seções, que:

$$
A=\lim _{n \rightarrow \infty} \frac{d\left(y_{0}, y_{n}\right)}{n}=\frac{1}{2}, \quad \text { para quase todo } \omega \in \Omega
$$

\subsection{Aplicando Teorema de Kingman a $d\left(y_{0}, y_{n}\right)$}

Podemos supor $y_{0}=e$, como visto na Proposição 2.13 que o drift não depende da escolha de $y_{0}$. Definimos $H: \mathbb{N} \times \Omega \rightarrow\{-1,1\}$ por:

$$
H(i, \omega):=\left\{\begin{array}{cl}
-1 & \text { se } N(i, \omega)=\omega_{i}^{-1} \\
1 & \text { caso contrário }
\end{array}\right.
$$

onde $N(i, \omega)$ é o primeiro elemento à direita da forma reduzida de $u(i, \omega)$ se $u(i, \omega) \neq e$ e $N(i, \omega):=e$ se $u(i, \omega)=e$. Observe que:

$$
d\left(y_{0}, y_{n}\right)=\sum_{i=1}^{n} H(i, \omega) \quad \text { para } n \geq 1 .
$$

Notemos que $H^{+}(0, \omega)=1$ é integrável em $\Omega$ e pela desigualdade triangular 
temos:

$$
\begin{aligned}
\sum_{i=1}^{n+m} H(i, \omega)=d\left(y_{0}, y_{n+m}\right) & \leq d\left(y_{0}, y_{n}\right)+d\left(y_{n}, y_{n+m}\right) \\
& =\sum_{i=1}^{n} H(i, \omega)+\sum_{i=1}^{m} H\left(i, T^{n} \omega\right)
\end{aligned}
$$

Pelo Teorema de Kingman 2.9, existe constante $A$ satisfazendo:

$$
A=\lim _{n \rightarrow \infty} \frac{1}{n} \sum_{i=1}^{n} \int_{\Omega} H(i, \omega) d \mu=\lim _{n \rightarrow \infty} \frac{1}{n} \sum_{i=1}^{n} H(i, \omega), \quad \text { para quase todo } \omega \in \Omega \text {. }
$$

Por outro lado, abreviando $N_{i}:=N(i, \omega)$, temos:

$$
\int_{\Omega} H(i, \omega) d \mu=\mu\left\{N_{i} \neq \omega_{i}^{-1}\right\}-\mu\left\{N_{i}=\omega_{i}^{-1}\right\}
$$

Mostraremos que o limite quando $i \rightarrow \infty$ do lado direito da equação acima é 1/2. Para isso, provaremos as duas afirmações da próxima seção.

\subsection{Duas afirmações para mostrar que $A=\frac{1}{2}$}

Afirmação 3.3. O lado direito da equação (3.3) é igual a: $\frac{1}{2}\left(1+\mu\left\{N_{i}=e\right\}\right)$.

Em particular, a afirmação implica que $A \geq \frac{1}{2}$.

Prova. Como $\left[N_{i} \neq \omega_{i}^{-1}\right]=\Omega \backslash\left[N_{i}=\omega_{i}^{-1}\right]$, o lado direito de (3.3) é igual a:

$$
\begin{aligned}
1-2 \mu\left\{N_{i}=\omega_{i}^{-1}\right\} & =1-2 \sum_{l \in\left\{a, b, a^{-1}, b^{-1}\right\}} \mu\left\{\left[N_{i}=l\right] \cap\left[\omega_{i}^{-1}=l\right]\right\} \\
& =1-2 \sum_{l \in\left\{a, b, a^{-1}, b^{-1}\right\}} \mu\left\{N_{i}=l\right\} \cdot \mu\left\{\omega_{i}=l\right\} \\
& =1-\frac{1}{2} \sum_{l \in\left\{a, b, a^{-1}, b^{-1}\right\}} \mu\left\{N_{i}=l\right\} \\
& =\frac{1}{2}\left(1+\mu\left\{N_{i}=e\right\}\right) .
\end{aligned}
$$

Na segunda equação acima usamos independência, na terceira linha usamos $\mu\left\{\omega_{i}=l\right\}=\frac{1}{4}$ e na última linha usamos:

$$
\mu\left\{N_{i}=e\right\}+\sum_{l \in\left\{a, b, a^{-1}, b^{-1}\right\}} \mu\left\{N_{i}=l\right\}=1
$$

Agora usaremos a afirmação anterior para provar a segunda afirmação: 
Afirmação 3.4. $\lim _{i \rightarrow \infty} \mu\left\{N_{i}=e\right\}=0$.

Prova. Nesta demonstração usaremos a notação: $g_{i}(\omega):=\frac{1}{i} d\left(y_{i}(\omega), y_{0}\right)$. Como vimos anteriormente, $g_{i}$ converge a $A$ para quase todo $\omega \in \Omega$. Como a medida $\mu$ é finita, temos $g_{i}$ converge a $A$ em medida, em particular temos:

$$
\lim _{i \rightarrow \infty} \mu\left\{\left|g_{i}-A\right|>\frac{A}{2}\right\}=0
$$

Notemos que $\left[N_{i}=e\right]=\left[g_{i}=0\right] \subset\left[\left|g_{i}-A\right|>\frac{A}{2}\right]$, daí a equação acima implica $\lim _{i \rightarrow \infty} \mu\left\{N_{i}=e\right\}=0$.

\subsection{Aplicação do Teorema 2.10}

Tomando limite $i \rightarrow \infty$ e usando Afirmação 3.4, da equação (3.3) obtemos:

$$
\lim _{i \rightarrow \infty} \int_{\Omega} H(i, \omega) d \mu=\lim _{i \rightarrow \infty} \frac{1}{2}\left(1+\mu\left\{N_{i}=e\right\}\right)=\frac{1}{2} .
$$

finalmente pela (3.2) concluímos:

$$
A=\lim _{n \rightarrow \infty} \frac{1}{n} \sum_{i=1}^{n} \int_{\Omega} H(i, \omega) d \mu=\frac{1}{2}
$$

Como o drift $A=\frac{1}{2}>0$. Pelo Teorema 2.10 para quase todo $\omega \in \Omega$ e para todo vértice $y_{0}$, existe um raio geodésico $\gamma$ de velocidade unitária no Grafo de Cayley com $\gamma(0)=y_{0}$ com de modo que:

$$
\lim _{n \rightarrow \infty} \frac{d\left(\gamma\left(\frac{n}{2}\right), y_{n}\right)}{n}=0
$$

Notemos que as geodésicas no $X$ são exatamente os caminhos de velocidade 1, que percorrem arestas e vértices do grafo "sem voltar". Também geodésica depende de $\omega$ qtp, é nesse aspecto que o Teorema de K-M diferencia do Teorema de Birkhoff, que diz que existe uma única geodésica em $\mathbb{R}$ aproximando as somas de Birkhoff para $\mu$-qtp $\omega$. 


\section{Segundo Exemplo: Isometrias do Disco Hiperbólico}

Definiremos um cociclo de isometrias sobre o disco unitário com a métrica hiperbólica. Demonstraremos que este satisfaz as hipóteses do Teorema de K-M 2.10 e sob certas condições (o "passo" de cada isometria é grande), mostremos que o drift $A>0$. Na prova faremos uma comparação com o cociclo na árvore apresentada no capítulo anterior. Veremos que essa comparação não é possível se os passos não forem suficientemente grandes.

\subsection{Definição do Cociclo de Isometrias}

Neste seção, algumas demonstrações de propriedades bem conhecidos foram omitidos. A maior parte dos conceitos omitidos que nesta seção pode ser encontrado em [Needham].

Definição 4.1. Definimos o disco hiperbólico $\mathbb{D} \subset \mathbb{R}^{2}$ como o conjunto:

$$
\mathbb{D}:=\left\{\left.z \in \mathbb{R}^{2}|| z\right|^{2}<1\right\}
$$

onde |.| é a norma euclidiana.

Dado $z \in \mathbb{D}$ consideremos a métrica $g_{z}: T_{z} \mathbb{D} \times T_{z} \mathbb{D} \rightarrow \mathbb{R}$ dada por:

$$
g_{z}(u, v):=\frac{\langle u, v\rangle}{\left(1-|z|^{2}\right)^{2}}
$$

O par $(\mathbb{D}, g)$ é uma variedade Riemanniana de curvatura seccional constante igual a -4 . Recordamos que o traço das geodésicas em $\mathbb{D}$ são ou segmento de retas passando pela origem ou arco de círculos ortogonais a fronteira $\partial \mathbb{D}$.

Denotamos por $d$ a distância hiperbólica induzida pela métrica $g$. Dado um ponto $z \in \mathbb{D}$, a distância hiperbólica está relacionada à distância Euclidiana pela igualdade $d(z, 0)=\tanh ^{-1}|z|$. Usaremos essa conversão com frequência ao longo do capítulo. 
Consideremos duas geodésicas $\alpha, \beta: \mathbb{R} \rightarrow \mathbb{D}$, de velocidades unitárias, dadas por:

$$
\begin{aligned}
\alpha(t) & :=\left(\tanh \left(\frac{t}{2}\right), 0\right), \\
\beta(t) & :=\left(0, \tanh \left(\frac{t}{2}\right)\right) .
\end{aligned}
$$

Geometricamente $\alpha$ e $\beta$ se cruzam perpendicularmente na origem no tempo $t=0$, e os seus traços coincidem com os segmentos dos eixos $x$ e $y$ do plano encontrados dentro do disco respectivamente.

Consideremos o grupo unitário especial:

$$
S U(1,1):=\left\{M \in \mathcal{M}_{2 \times 2}(\mathbb{C}) \mid M=\left(\begin{array}{cc}
a & b \\
\bar{b} & \bar{a}
\end{array}\right), \operatorname{det} M=1\right\} .
$$

Os elementos $M \in S U(1,1)$ podem ser associados a transformação de Möbius $I_{M}: \mathbb{D} \rightarrow \mathbb{D}$ através da identificação:

$$
M=\left(\begin{array}{cc}
a & b \\
\bar{b} & \bar{a}
\end{array}\right) \quad \forall z \in \mathbb{D} \longmapsto I_{M}(z)=\frac{a z+b}{\bar{b} z+\bar{a}} .
$$

A transformação $I_{M}$ é isometria de $(\mathbb{D}, d)$. Além disso, a associação $M \in$ $S U(1,1) \mapsto I_{M} \in I s o(\mathbb{D})$ é um homomorfismo de grupos.

A cada $l \in\left(0, \frac{1}{2}\right)$, definimos as isometrias $I_{1, l}, I_{2, l}$ associada aos matrizes $M_{1, l}, M_{2, l}$, respectivamente, dadas por:

$$
\begin{aligned}
& M_{1, l}:=\frac{1}{1-l^{2}}\left(\begin{array}{cc}
1+l^{2} & 2 l \\
2 l & 1+l^{2}
\end{array}\right), \\
& M_{2, l}:=\frac{1}{1-l^{2}}\left(\begin{array}{cc}
1+l^{2} & 2 l i \\
-2 l i & 1+l^{2}
\end{array}\right) .
\end{aligned}
$$

Geometricamente $I_{1, l}$ preserva a geodésica $\alpha$ e envia o ponto $(-l, 0)$ em $(l, 0)$. Portanto $I_{1, l}$ translada a geodésica $\alpha$ por uma distância hiperbólica $2 \tanh ^{-1} l$ para direita. Analogamente, $I_{2, l}$ translada a geodésica $\beta$ por uma distância hiperbólica de $2 \tanh ^{-1} l$ para cima.

Consideremos quatro geodésicas $\alpha_{1}, \beta_{2}, \alpha_{2}, \beta_{1}$ "simétricas entre si" no seguinte sentido. As geodésicas $\alpha_{1}$ e $\alpha_{2}$ interceptam o eixo $y$ ortogonalmente nos pontos $(0,-l)$ e $(0, l)$ respectivamente. Da mesma forma, as geodésicas $\beta_{1}$ e $\beta_{2}$ interceptam o eixo $x$ ortogonalmente nos pontos $(-l, 0)$ e $(l, 0)$ 


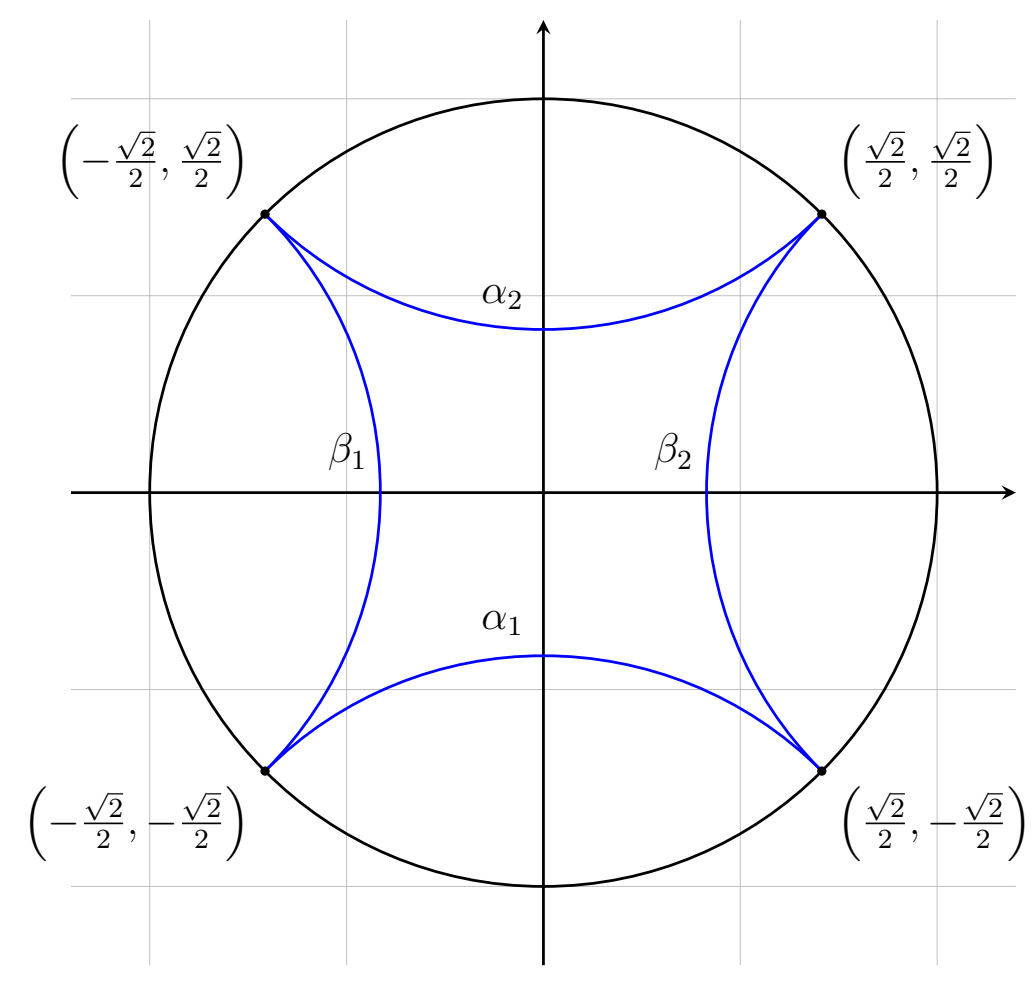

Figura 4.1: As quatro geodésicas são arcos dos círculos tangentes entre si, quando $l=\sqrt{2}-1$.

respectivamente. Segue da definição que:

$$
\begin{aligned}
& I_{1}\left(\beta_{1}\right)(t)=\beta_{2}(t), \\
& I_{2}\left(\alpha_{1}\right)(t)=\alpha_{2}(t) .
\end{aligned}
$$

A Figura 4.1 mostra um caso particular onde $l=\sqrt{2}-1$, as quatro geodésicas são "tangentes" entre si no bordo do disco $\partial \mathbb{D}$ :

Definimos também $I_{3, l}:=I_{1, l}^{-1}, I_{4, l}:=I_{2, l}^{-1}$. Quando não há ambiguidade sobre o valor de $l$ usaremos simplesmente $I_{1}, I_{2}, I_{3}, I_{4}$ para denotar as isometrias.

Seja $G$ o grupo gerado pelas isometrias $I_{1}, I_{2}$ e representamos o elemento neutro usando $i d$. Definimos $\Omega:=\{1,2,3,4\}^{\mathbb{N}}$, com a medida de Bernoulli com pesos $1 / 4$. Definimos o cociclo $u: \mathbb{N} \times \Omega \rightarrow G$ onde:

$$
u(n, \omega)= \begin{cases}I_{\omega_{0}} \circ I_{\omega_{1}} \circ \ldots \circ I_{\omega_{n-1}} & \text { se } n \geq 1 \\ i d & \text { se } n=0\end{cases}
$$

Seja $T: \Omega \rightarrow \Omega$ o shift para esquerda, que é uma transformação preservando a medida e ergódica. 


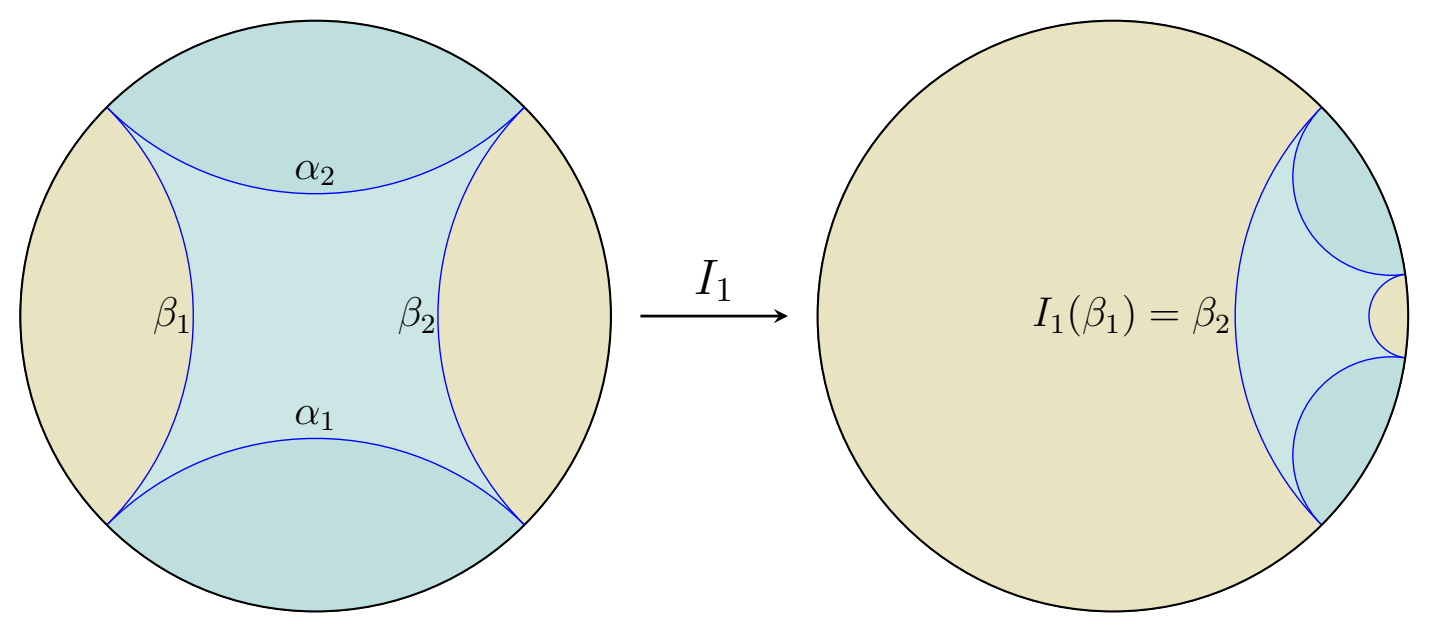

Figura 4.2: A figura acima mostra para o caso em que $l=\sqrt{2}-1$. Quando $l>\sqrt{2}-1$ (cf. Fig. 4.3) as geodésicas se afastam mais entre si, por isso as regiões $R_{1}, R_{2}, R_{3}$, $R_{4}$ são dois-a-dois disjuntos.

\subsection{Propriedades do Cociclo $u$}

Começamos introduzindo notações que serão utilizadas nesta seção. Vamos considerar as regiões abertas:

$R_{1}$ a região à direita de $\beta_{2}, S_{1}$ a região à esquerda de $\beta_{2}$,

$R_{2}$ a região acima de $\alpha_{2}, S_{2}$ a região abaixo de $\alpha_{2}$,

$R_{3}$ a região à esquerda de $\beta_{1}, S_{3}$ a região à direita de $\beta_{1}$,

$R_{4}$ a região aberta abaixo de $\alpha_{1}, S_{4}$ a região acima de $\alpha_{1}$.

Introduzimos a notação no conjunto $\{1,2,3,4\}$ colocando $\overline{1}:=3, \overline{2}:=4, \overline{3}:=1$ e $\overline{4}:=2$.

Observe que se $l=\sqrt{2}-1$, a isometria $I_{1}$ é da forma mostrada na Figura 4.2 .

Quando $l \geq \sqrt{2}-1$, temos região $S_{3}$ é enviada pela aplicação $I_{1}$ para dentro da região $R_{1}$, isto é, $I_{1}\left(S_{3}\right) \subset R_{1}$. Analogamente ocorre para outras três pares regiões. Em geral temos $I_{i}\left(S_{\bar{i}}\right) \subset R_{i}$. Como as quatro regiões $R_{1} \cap R_{2} \cap R_{3} \cap R_{4}=\emptyset$ são disjuntos quando $l \geq \sqrt{2}-1$, segue que o conjunto:

$$
\bigcup_{\substack{\omega \in \Omega \\ n \in \mathbb{N}}}(u(n, \omega)(\alpha)) \cup(u(n, \omega)(\beta))
$$

é simplesmente conexo (cf. Fig. 4.5), e veremos mais em diante que o conjunto acima é um grafo de Cayley.

Proposição 4.2. Se $l \geq \sqrt{2}-1$ então $G$ é grupo livre. 


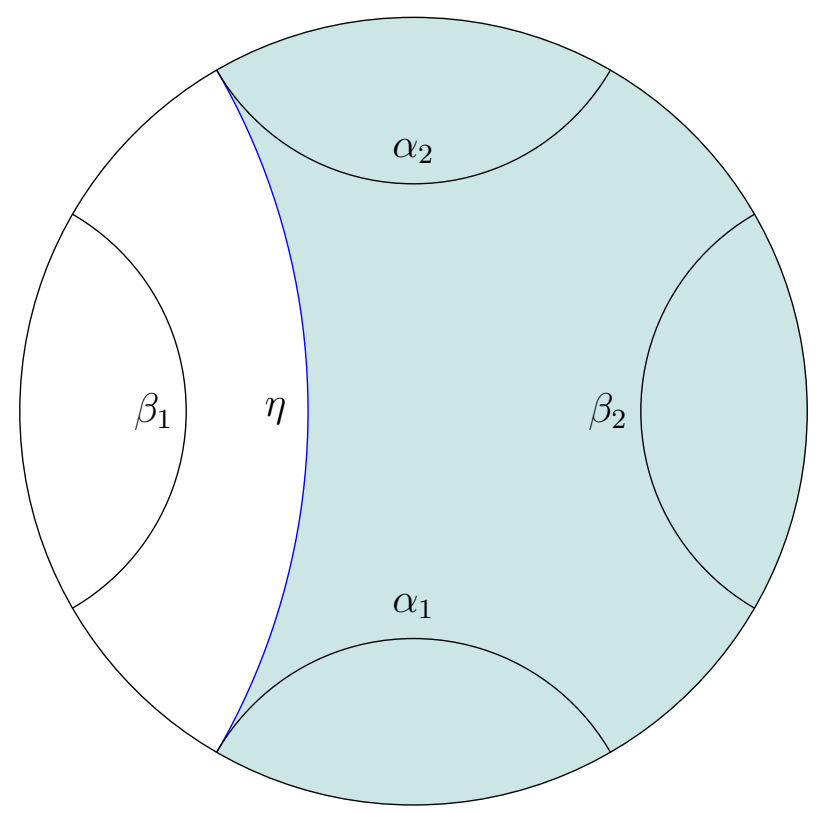

Figura 4.3: A geodésica $\eta$ "tangência" $\alpha_{1}$ e $\alpha_{2}$ nos pontos mostrados e intercepta $\alpha$ perpendicularmente.

Prova. Sejam $I_{i_{1}}, \ldots, I_{i_{n}}$ tais que $i_{j+1} \neq \bar{i}_{j}$ para todo $j \in\{1, \ldots, n-1\}$. Seja $J:=I_{i_{n}} \circ \cdots \circ I_{i_{1}}$. Segue dos argumentos anteriores que $J\left(S_{i_{1}}\right) \subset R_{i_{n}}$ portanto $J \neq i d$.

Proposição 4.3. Se $l>\sqrt{2}-1$ então existe um constante $K(l)>0$ tal que para todo $i \in\{1,2,3,4\}$ vale:

$$
d\left(I_{i}(y), 0\right) \geq d(y, 0)+K \quad \text { para todo } y \in R_{j}, \operatorname{com} j \neq \bar{i}
$$

Prova. Provaremos a proposição apenas para $i=1$, pois para outros $i$ 's a prova é análoga. Antes de começar, consideremos $\tilde{S}_{3}$ a região aberta à direita da geodésica $\eta$ tangenciando $\alpha_{1}$ e $\alpha_{2}$ da maneira como mostra na Figura 4.3

Seja $z \in \mathbb{D}$ o ponto de intersecção da geodésica $\eta \operatorname{com} \alpha$. Chamamos:

$$
\delta=\tanh ^{-1} l-d(z, 0)
$$

Observe também que $R_{1} \cup R_{2} \cup R_{4} \subset \tilde{S}_{3}$. Seja $y \in \tilde{S}_{3}$, vamos mostrar que vale (4.2).

Caso 1: se $y \in \alpha$, por definição de $I_{1}$ e equação (4.3):

$$
d\left(I_{1}(y), 0\right)>d(y, 0)+2 \delta .
$$

Caso 2: se $y \notin \alpha$, existe um único ponto $x \in \alpha \cap \tilde{S}_{3}$ tal que a geodésica passando por $y$ e $x$ é perpendicular a $\alpha$ (cf. Fig. 4.4): 


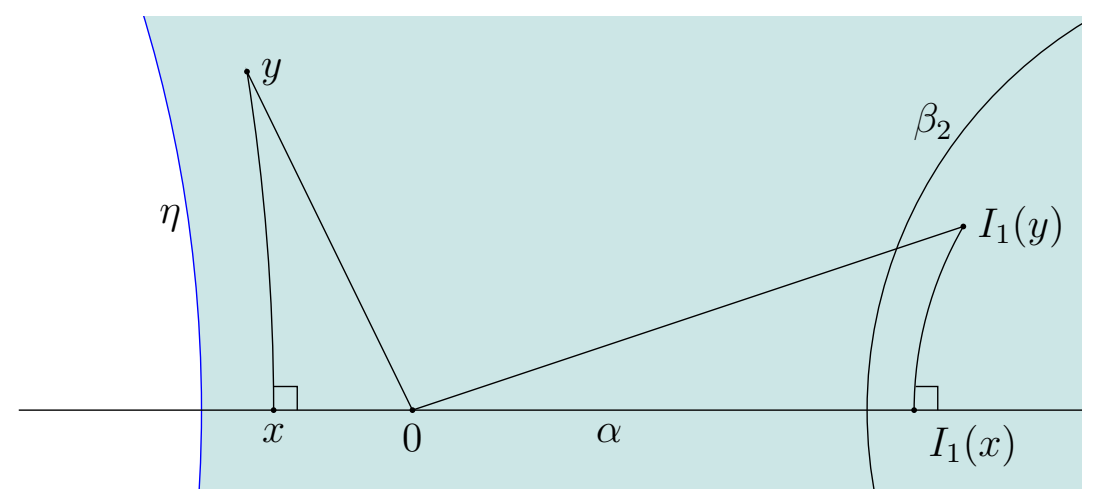

Figura 4.4: Um esboço da localização dos pontos e das geodésicas.

A isometria $I_{1}$ leva o segmento $\overline{x y}$ ao segmento $\overline{I_{1}(x) I_{1}(y)}$, portanto este é perpendicular ao $\alpha$ também. Note que os triângulos $\triangle 0 x y$ e $\triangle 0 I_{1}(x) I_{1}(y)$ são triângulos retângulos. Pelo Teorema de Pitágoras Hiperbólico aplicado a estes dois triângulos, temos:

$$
\begin{aligned}
& \cosh t_{1}=\cosh d(x, y) \cosh s_{1}, \\
& \cosh t_{2}=\cosh d\left(I_{1}(x), I_{1}(y)\right) \cosh s_{2},
\end{aligned}
$$

onde $t_{1}=d(y, 0), t_{2}=d\left(I_{1}(y), 0\right), s_{1}=d(x, 0)$ e $s_{2}=d\left(I_{1}(x), 0\right)$. Como $d(x, y)=d\left(I_{1}(x), I_{1}(y)\right)$, usando as duas equações acima e tomando logaritmo, obtemos:

$$
\begin{aligned}
\cosh t_{1} \cosh s_{2} & =\cosh t_{2} \cosh s_{1} \\
\Longrightarrow \log \left(\cosh t_{2}\right)-\log \left(\cosh t_{1}\right) & =\log \left(\cosh s_{2}\right)-\log \left(\cosh s_{1}\right) .
\end{aligned}
$$

Pelo Caso 1 temos $s_{2}-s_{1}>2 \delta$. Notemos que a função $f(t)=\log (\cosh t)$ tem a derivada $f^{\prime}(t)=\tanh t$ e $f^{\prime \prime}(t)=\frac{1}{\cosh ^{2} t}$ e observe que $f^{\prime}(t), f^{\prime \prime}(t)>0$ em $(0, \infty)$. Da convexidade de $f$ temos:

$$
f\left(s_{2}-s_{1}\right)-f(0)=\int_{0}^{s_{2}-s_{1}} f^{\prime}(t) d t \leq \int_{0}^{s_{2}-s_{1}} f^{\prime}\left(t+s_{1}\right) d t=f\left(s_{2}\right)-f\left(s_{1}\right) .
$$

Portanto pela equação (4.4) e a desigualdade acima temos:

$$
f(2 \delta)<f\left(s_{2}-s_{1}\right)-f(0) \leq f\left(s_{2}\right)-f\left(s_{1}\right)=f\left(t_{2}\right)-f\left(t_{1}\right) .
$$

Mas $0<f^{\prime}(t)<1$ em $(0, \infty)$, concluímos $t_{2}-t_{1}>f\left(t_{2}\right)-f\left(t_{1}\right)>f(2 \delta)$, onde $f(2 \delta)>0$ depende apenas de $l$. Tomamos $K(l):=f(2 \delta)$ e recapitulando as definições de $t_{1}$ e $t_{2}$, a proposição está provada.

Observação. Na demonstração, o ponto $z=\left(-\frac{1-l}{1+l}, 0\right)$, e através de uma 


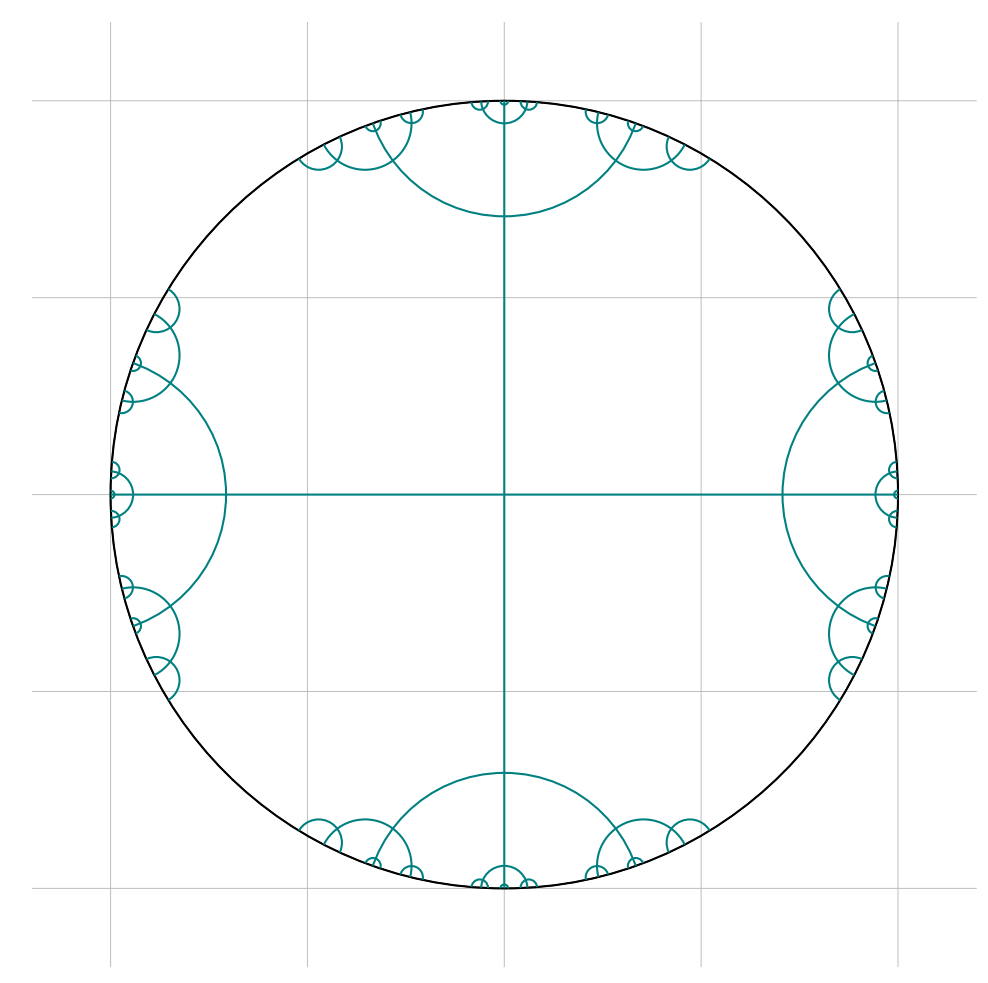

Figura 4.5: Quando $l \geq \sqrt{2}-1$ o conjunto (4.1) é um grafo de Cayley no disco Hiperbólico.

conta simples, é fácil conferir $\delta=\frac{1}{2} \log \frac{l(1+l)}{1-l}$.

\subsection{Aplicação do Teorema 2.10}

Considerando a árvore $X$ cujos vértices são os pontos de todas as órbitas possíveis de $y_{0}=0$ e arestas como os segmentos das geodésicas ligando dois vértices $p, q$ de modo $I_{k}(p)=q$ para algum $k \in\{1,2,3,4\}$ (cf. Fig. 4.5). Cada aresta pode ser parametrizada pelo intervalo $[0,1]$ de maneira proporcional à métrica hiperbólica. Como na Seção 3.1, fica determinada uma distância $\tilde{d}$ no grafo, tal que a distância entre dois vértices $p$ e $q$ é:

$$
\tilde{d}(p, q):=\min _{\text {c caminho de } \mathrm{p} \text { ao } \mathrm{q}}[\text { número de arestas de } \mathrm{c}] .
$$

O par $(X, \tilde{d})$ forma um espaço métrico, e $u$ induz de forma natural um cociclo de isometrias $\tilde{u}$ em $X$. A Proposição 4.3 mostra que se $y \in X$ é um vértice na região $R_{j}$, então:

$$
d\left(I_{i}(y), 0\right) \geq d(y, 0)+K \quad \text { para todo } i \in\{1,2,3,4\} \backslash \bar{j} .
$$

Usando indução, temos:

$$
d(u(n, \omega) 0,0) \geq K \tilde{d}(u(n, \omega) 0,0) \quad \text { para todo } n \in \mathbb{N} .
$$


Pela equação do "drift" do cociclo na árvore (3.1) temos:

$$
A=\lim _{n \rightarrow \infty} \frac{d(u(n, \omega) 0,0)}{n} \geq \lim _{n \rightarrow \infty} \frac{K \tilde{d}\left(y_{n}, 0\right)}{n}=\frac{1}{2} K>0
$$

para quase todo $\omega \in \Omega$. Isso mostra que o drift $A$ é positivo quando $l>\sqrt{2}-1$. Substituindo o valor de $K$ obtido na prova da Proposição 4.3, temos:

Proposição 4.4. Se $l>\sqrt{2}-1$, então:

$$
A \geq \frac{1}{2} \log \left(\cosh \left(\log \frac{l(1+l)}{1-l}\right)\right)>0 .
$$

Pelo Teorema K-M, quando $l>\sqrt{2}-1$ para quase todo $\omega \in \Omega$ e para todo $p \in \mathbb{D}$ existe uma geodésica hiperbólica $\gamma$ de velocidade unitária começando em $p$ tal que:

$$
\lim _{n \rightarrow \infty} \frac{d(u(n, \omega) p, \gamma(t A))}{n}=0 .
$$

Isso é a propriedade de rastreamento "sublinear" para o cociclo $u$.

Para garantir que o drift $A>0$, a condição de $l>\sqrt{2}-1$ pode ser enfraquecida. Posteriormente, na Seção 5.4 mostraremos que $A>0$ para qualquer $l \in\left(0, \frac{1}{2}\right)$. Para isso, usaremos os Teoremas de Oseledets e de Furstenberg.

\subsection{Comportamento no caso $\omega=(\overline{1234})$}

Observando a figura do grafo de Cayley dentro do disco hiperbólico, a trajetória $u(n, \omega) 0$ para $\omega=(\overline{1234})$ é dentre as sequências onde não há cancelamentos, é uma das que mais demora para chegar ao bordo do disco, isto é, que se afasta mais lentamente da posição original. De fato, a velocidade em que $u(n, \omega) 0$ aproxima do bordo depende do valor de $l$. Mostraremos isso nessa seção.

Ao longo da seção iremos considerar $\omega:=(\overline{1234})$, definimos a matriz $M:=M_{1} M_{2} M_{3} M_{4}$, seja $T$ a transformação de Möbius associada a $M$ e denotamos $y_{n}:=u(n, \omega) 0$. Logo $y_{4 n}=T^{n}(0)$ e e um cálculo direto mostra que:

$$
M=\left(\frac{1}{1-l^{2}}\right)^{4}\left(\begin{array}{cc}
\left(a^{2}-b^{2} i\right)^{2}-2 a^{2} b^{2} & 2 a b^{3}(-1+i) \\
2 a b^{3}(-1-i) & \left(a^{2}+b^{2} i\right)^{2}-2 a^{2} b^{2}
\end{array}\right)
$$




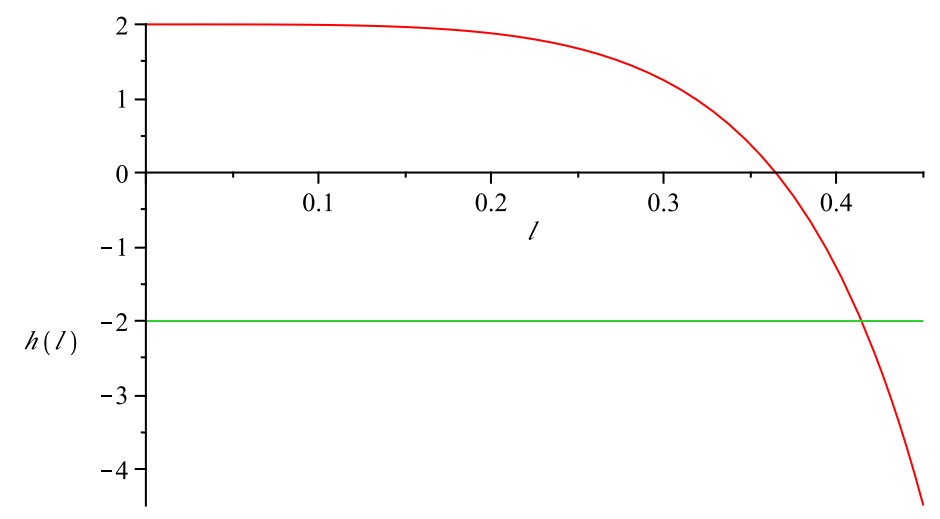

Figura 4.6: Gráfico de $h$ em função de $l$. Temos $h\left(l_{0}\right)=0 \operatorname{com} l_{0}=\left(2^{\frac{1}{2}}+1\right)^{\frac{1}{2}}-2^{\frac{1}{4}}$. onde $a=1+l^{2}$ e $b=2 l$. Daí o traço de $M$ é dado pela fórmula:

$$
\operatorname{traço}(M)=2-4\left(\frac{2 l}{1-l^{2}}\right)^{4} .
$$

Definimos a função $h(l):=\operatorname{traço}(M)$ para $0<l<\frac{1}{2}$. É imediato que $h$ é decrescente (cf. Fig. 4.6). Após uma conta direta, conclui-se que (cf. [Needham, p.170]):

$$
\begin{array}{clll}
T \text { é hiperbólico } & \Leftrightarrow|h(l)|>2 & \Leftrightarrow l>\sqrt{2}-1 . \\
T \text { é parabólico } & \Leftrightarrow|h(l)|=2 \quad \Leftrightarrow \quad l=\sqrt{2}-1 . \\
T \text { é elíptico } & \Leftrightarrow|h(l)|<2 \quad \Leftrightarrow \quad l<\sqrt{2}-1 .
\end{array}
$$

Usando a propriedades acima, distância hiperbólica e levando em conta que $T$ fixa $\mathbb{D}$ :

$$
\begin{aligned}
& l>\sqrt{2}-1 \Rightarrow T \text { tem } 2 \text { pontos fixos na } \partial \mathbb{D} \text { e } \lim _{n \rightarrow \infty} \frac{1}{n} d_{n}>0 . \\
& l=\sqrt{2}-1 \Rightarrow T \text { tem } 1 \text { ponto fixo na } \partial \mathbb{D}, \lim _{n \rightarrow \infty} \frac{1}{n} d_{n}=0 \text { e } \lim _{n \rightarrow \infty} d_{n}=\infty . \\
& l<\sqrt{2}-1 \Rightarrow T \text { tem } 1 \text { ponto fixo no } \mathbb{D} \text { e }\left\{d_{n}\right\}_{n \in \mathbb{N}} \text { é limitada. }
\end{aligned}
$$

Onde $d_{n}:=d\left(y_{n}, 0\right)$. Os dados acima mostra que não podemos comparar a distância do grafo com a distância no disco quando $l \leq \sqrt{2}-1$, já que no grafo temos $\lim _{n \rightarrow \infty} \frac{1}{n} \tilde{d}_{n}\left(y_{n}, 0\right)=\frac{1}{2}>0$. Geometricamente, os grafos gerados pelas isometrias têm auto-intersecções quando $l<\sqrt{2}-1$. E no caso $l=\sqrt{2}-1$ apesar do grafo ser uma árvore, existem vários $\omega$ 's periódicos cujas trajetórias descrita por $y_{n}$ são parabólicas, e portanto estes têm drift $A(\omega)$ nulo. 


\section{Teorema de Oseledets}

Apresentaremos uma demonstração do Teorema de Oseledets 5.2 pela aplicação do Teorema de K-M 2.10. Depois apresentaremos um exemplo de cociclo de matrizes $2 \times 2$ que possui dois expoentes de Lyapunov distintos, e como aplicação desse mesmo exemplo, generalizaremos o resultado de $A>0$ para $l>\sqrt{2}-1$ do Capítulo 4 .

\subsection{Teorema de Oseledets}

Ao longo do capítulo consideremos $(\Omega, \mathcal{A}, \mu)$ um espaço de medida, $T: \Omega \rightarrow \Omega$ uma transformação ergódica. $G L_{k}(\mathbb{R})$ indica o subgrupo das matrizes $k \times k$ com determinante não nulo.

Definimos a norma de uma matriz $M \in \mathcal{M}_{k \times k}(\mathbb{R})$ como:

$$
\|M\|:=\sup _{\|v\|=1}\|M v\|, \quad \text { onde } v \in \mathbb{R}^{k} .
$$

Quando $M$ é simétrica, a sua norma coincide com o maior autovalor de $M$. Ao longo do capítulo $I$ denotará matriz identidade.

Definição 5.1 (cociclo à esquerda). Dizemos que a aplicação $u: \mathbb{N} \times \Omega \rightarrow$ $G$ é cociclo à esquerda quando u satisfaz:

1. $u(0, \omega)=e \quad$ onde e é o elemento neutro de $G$,

2. $u(n+m, \omega)=u\left(m, T^{n} \omega\right) * u(n, \omega) \quad \forall n, m \in \mathbb{N}$.

Teorema 5.2. Seja $M: \mathbb{N} \times \Omega \rightarrow G L_{k}(\mathbb{R})$ um cociclo à esquerda tal que:

$$
\int_{\Omega} \log \max \left\{\|M(1, \omega)\|,\left\|M(1, \omega)^{-1}\right\|\right\} d \mu<\infty
$$

Então existem os valores $\lambda_{1}>\ldots>\lambda_{l}$, chamados de expoentes de Lyapunov, tais que para quase todo $\omega \in \Omega$ existe uma bandeira de subespaços:

$$
\mathbb{R}^{k}=V_{1}(\omega) \supset \ldots \supset V_{l}(\omega) \supset V_{l+1}(\omega)=\{0\}
$$


tais que para todo $v \in \mathbb{R}^{k} \backslash\{0\}$

$$
v \in V_{i} \backslash V_{i-1} \Longleftrightarrow \lambda_{i}=\lim _{n \rightarrow \infty} \frac{1}{n} \log \frac{\|M(n, \omega) v\|}{\|v\|}
$$

para todo $i \in\{1, \ldots, l\}$. Além disso, $\lambda_{i} \circ T=\lambda_{i}$.

Apresentaremos uma demonstração de Teorema de Oseledets usando o Teorema de K-M 2.10.

\section{2 $\operatorname{Pos}_{k}(\mathbb{R})$ como uma Variedade Riemanniana}

Este seção é preliminar para a demonstração do Teorema de Oseledets. Mencionaremos, sem provar, vários propriedades de $\mathrm{Pos}_{k}$ como uma variedade Riemanniana. Essas propriedades podem ser encontradas em [Lang, p.322$338]$.

Definimos o conjunto das matrizes simétricas e matrizes positivas (definidas) como:

$$
\begin{gathered}
\operatorname{Sym}_{k}:=\left\{X \in \mathcal{M}_{k \times k}(\mathbb{R}) \mid X=X^{*}\right\} \\
\operatorname{Pos}_{k}:=\left\{P \in \text { Sym }_{k} \mid \text { todos os autovalores de } P \text { são positivos }\right\} .
\end{gathered}
$$

Definimos uma relação de semi-ordem em $S_{\text {ym }}$. Sejam $X, Y \in S y m_{k}$; então dizemos $X \leq Y$ se e somente se $\langle X v, v\rangle \leq\langle Y v, v\rangle$ para todo $v \in R^{k}$.

O $S_{y m_{k}}$ é um espaço vetorial, e o $\operatorname{Pos}_{k}$ é um subconjunto aberto de Sym $_{k}$. Portanto, para toda matriz $P \in P_{o s}$, podemos identificar o plano tangente $T_{P} P_{o s}$ com $S_{y m}$. Definimos a métrica em $\operatorname{Pos}_{k}$ :

$$
\langle X, Y\rangle_{P}=\operatorname{tr}\left(P^{-1} X P^{-1} Y\right), \quad \forall X, Y \in \operatorname{Sym}_{k}
$$

$\langle., .\rangle_{P}$ é chamada de métrica traço. Seja $d$ a distância Riemanniana induzida, temos a seguinte fórmula para a distância.

Teorema 5.3. Sejam P, $Q \in \operatorname{Pos}_{k}$. Sejam $a_{1}, \ldots, a_{k}$ raízes do polinômio $\operatorname{det}(t P-Q)$ contando com multiplicidade. Então:

$$
d(P, Q)=\left(\sum_{i=1}^{k}\left(\log a_{i}\right)^{2}\right)^{\frac{1}{2}}
$$

Convém complementar que as raízes de $\operatorname{det}(t P-Q)$ são os autovalores $d a$ matriz $P^{-\frac{1}{2}} Q P^{-\frac{1}{2}}$.

Nessa métrica, o $\mathrm{Pos}_{k}$ é completo, simplesmente conexo e tem as curva- 
turas seccionais não-positivas. Portanto segue da Proposição 6.2 que $\operatorname{Pos}_{k}$ é Busemann NPC e uniformemente convexo. Dados $P \in P_{o s}$ e $t \in \mathbb{R}$, podemos diagonalizar $P=R D R^{*} \operatorname{com} D=\operatorname{diag}\left(a_{1}, \ldots, a_{k}\right)$ e $R$ é matriz ortogonal. Definimos $t$-ésima potência de $P$ como $P^{t}:=R D^{t} R^{*}$ onde $D^{t}:=\operatorname{diag}\left(a_{1}^{t}, \ldots, a_{k}^{t}\right)$.

As geodésicas $\gamma$ em $\operatorname{Pos}_{k} \operatorname{com} \gamma(0)=I$ são da forma $\gamma(t)=e^{X t}$ onde $X \in$ Sym $_{k}$. Portanto as geodésicas podem ser escritas na forma $Q^{\frac{1}{2}} P^{2 t} Q^{\frac{1}{2}}$, onde $Q \in P o s_{k}$ é a matriz posição da geodésica em $t=0$.

Definição 5.4 (ação de $G L_{k}(\mathbb{R})$ em $\operatorname{Pos}_{k}$ ). A cada $M \in G L_{k}(\mathbb{R})$ associamos a uma aplicação $[M]: \operatorname{Pos}_{k} \rightarrow$ Pos $_{k}$ dada por:

$$
[M] P=M P M^{*}
$$

Note que $[M]: \operatorname{Pos}_{k} \rightarrow \operatorname{Pos}_{k}$ é isometria e preserva a relação de semiordem* $^{*}$ mencionada. A ação também é transitiva, de fato, dados $P, Q \in P_{o s}$, temos $\left[Q^{\frac{1}{2}} P^{-\frac{1}{2}}\right] P=Q$. Além disso, a associação $M \in G L_{k}(\mathbb{R}) \mapsto[M] \in$ $I s o\left(P_{k}\right)$ é um homomorfismo de grupos, pois $[M N]=[M] \circ[N]$. Com isso provaremos:

Proposição 5.5. Para todo $P, Q \in$ Pos $_{k}$ temos:

$$
e^{-d(P, Q)} P \leq Q \leq e^{d(P, Q)} P
$$

Prova. Sejam $a_{1}, \ldots, a_{d}$ os autovalores da matriz $\left[P^{-\frac{1}{2}}\right] Q$. Então pelo Teorema 5.3 temos:

$$
\left|\log a_{i}\right| \leq d\left(\left[P^{-\frac{1}{2}}\right] Q, I\right)=d(P, Q)
$$

Daí por definição da semi-ordem em $\operatorname{Pos}_{d}$, temos:

$$
e^{-d(P, Q)} I \leq\left[P^{-\frac{1}{2}}\right] Q \leq e^{d(P, Q)} I .
$$

Como a relação de semi-ordem é invariante pela ação, aplicando $\left[P^{\frac{1}{2}}\right]$ nas desigualdades acima, obtemos:

$$
e^{-d(P, Q)} P \leq Q \leq e^{d(P, Q)} P
$$

*Isto é, $P \leq Q$ se, e somente se $[M] P \leq[M] Q$. 


\subsection{Demonstração do Teorema de Oseledets}

Seja $M$ um cociclo à esquerda satisfazendo as hipóteses do Teorema 5.2. Definimos a função $U: \mathbb{N} \times \Omega \rightarrow I s o\left(P o s_{k}\right)$ associado ao cociclo à esquerda $M$ por $U(n, \omega)(P)=\left[M(n, \omega)^{*}\right] P$. Verificamos abaixo que $U$ é um cociclo à direita:

$$
\begin{aligned}
U(m+n, \omega) P & =\left[M(m+n, \omega)^{*}\right] P=\left[M(n, \omega)^{*} M\left(m, T^{n} \omega\right)^{*}\right] P \\
& =\left[M(n, \omega)^{*}\right] \circ\left[M\left(m, T^{n} \omega\right)^{*}\right] P \\
& =U(n, \omega) \circ U\left(m, T^{n} \omega\right)(P) .
\end{aligned}
$$

Dizemos que $U$ é o cociclo associado ao cociclo à esquerda $M$. Antes de iniciar a prova do Teorema de Oseledets, começamos mostrando dois lemas.

Lema 5.6. A condição de integrabilidade (5.1) para $M$ implica a condição momento finito (2.7) para $U$ do Teorema de $K-M$.

Prova. Tomemos $I \in \operatorname{Pos}_{k}$ e definimos $a_{1}(\omega), \ldots, a_{k}(\omega)$ os autovalores contando as multiplicidades de $U(1, \omega) I$. Observe que:

$$
\begin{aligned}
d(I, U(1, \omega) I) & =\left(\sum_{i=1}^{k}\left(\log a_{i}(\omega)\right)^{2}\right)^{\frac{1}{2}} \\
& \leq \sqrt{k} \log \max \left\{\|U(1, \omega) I\|,\left\|(U(1, \omega) I)^{-1}\right\|\right\} \\
& \leq 2 \sqrt{k} \log \max \left\{\|M(1, \omega)\|,\left\|M(1, \omega)^{-1}\right\|\right\}
\end{aligned}
$$

Na última desigualdade acima usamos $\|U(1, \omega) I\|=\left\|M(1, \omega) M(1, \omega)^{*}\right\| \leq$ $\|M(1, \omega)\|^{2}$. Integrando em ambos lados da desigualdade acima, obtemos:

$$
\int_{\Omega} d(I, U(1, \omega) I) d \mu \leq 2 \sqrt{k} \int_{\Omega} \log \max \left\{\|M(1, \omega)\|,\left\|M(1, \omega)^{-1}\right\|\right\} d \mu<\infty
$$

que é a condição (2.7) do Teorema de K-M.

O Lema 5.6 permite aplicar o Teorema de K-M 2.10 ao cociclo $U$, faremos isso posteriormente na demonstração do Teorema de Oseledets. Antes disso, mostraremos um lema técnico:

Lema 5.7. Sejam P, Q $\in$ Pos $_{k}$ tais que

$$
\lim _{n \rightarrow \infty} \frac{1}{n} d\left(P^{2 n},[M] Q^{2 n}\right)=0,
$$


para algum $M \in G L_{k}(\mathbb{R})$. Então $P$ e $Q$ são conjugadas, em particular possuem os mesmos autovalores contando as multiplicidades.

Uma interpretação geométrica da equação (5.4), é que $P^{2 t}$ e $[M] Q^{2 t}$ (com $t \in[0, \infty))$ são raios geodésicos que se mantém sublinearmente próximas.

Prova. Como $Q$ é diagonalizável, existem $R$ matriz ortogonal e $D=$ $\operatorname{diag}\left(a_{1}, \ldots, a_{k}\right)$, com $a_{1} \geq \ldots \geq a_{k}>0$, tais que $Q=[R] D$. Podemos fatorar a matriz $M R$ de forma única $M R=S U$, onde $S$ é ortogonal e $U$ é matriz triangular superior com entradas positivas na diagonal. Definimos a matriz $\tilde{P}:=[S] D \in \operatorname{Pos}_{k}$. Temos:

$$
d\left(\tilde{P}^{2 n},[M] Q^{2 n}\right)=d\left([S] D^{2 n},[M R] D^{2 n}\right)=d\left(I,\left[D^{-n} U\right] D^{2 n}\right) .
$$

Usamos $u_{i j}$ para denotar as entradas de $U$. Notemos que cada entrada da matriz $\left[D^{-n} U\right] D^{2 n}$ é:

$$
\sum_{h \geq \max (i, j)}\left(a_{i}^{-n} u_{i h}\right) a_{h}^{2 n}\left(a_{j}^{-n} u_{j h}\right)
$$

Observe que $h \geq \max (i, j)$ implica que $a_{h} \leq \min \left(a_{i}, a_{j}\right)$ e isso mostra que $a_{i}^{-n} a_{h}^{2 n} a_{j}^{-n} \leq 1$. Portanto cada entrada de $\left[D^{-n} U\right] D^{2 n}$ é limitada:

$$
\left|\sum_{h \geq \max (i, j)}\left(a_{i}^{-n} u_{i h}\right) a_{h}^{2 n}\left(a_{j}^{-n} u_{j h}\right)\right| \leq k \max _{h, l}\left\{\left|u_{h l}\right|\right\}^{2} .
$$

Usando a equivalência das normas em $\mathcal{M}_{k \times k}(\mathbb{R})$ temos $d\left(I,\left[D^{-n} U\right] D^{2 n}\right)$ é limitada independentemente de $n \in \mathbb{N}$. Portanto:

$$
\lim _{n \rightarrow \infty} \frac{1}{n} d\left(\tilde{P}^{2 n},[M] Q^{2 n}\right)=0 .
$$

Pela desigualdade triangular:

$$
\lim _{n \rightarrow \infty} \frac{1}{n} d\left(P^{2 n}, \tilde{P}^{2 n}\right) \leq \lim _{n \rightarrow \infty} \frac{1}{n} d\left(P^{2 n},[M] Q^{2 n}\right)+\lim _{n \rightarrow \infty} \frac{1}{n} d\left(\tilde{P}^{2 n},[M] Q^{2 n}\right)=0 .
$$

Como $\mathrm{Pos}_{k}$ tem curvatura não-positiva:

$$
d(P, \tilde{P}) \leq \frac{1}{2 n} d\left(P^{2 n}, \tilde{P}^{2 n}\right)
$$

Tomando limite $n \rightarrow \infty$ na equação acima, concluímos que $d(P, \tilde{P})=0$. Portanto $P=\tilde{P}$, que é conjugada a $Q$. 
Prova do Teorema de Oseledets. Aplicando o Teorema de K-M 2.10 ao cociclo de isometrias $U$ aplicado no ponto $Q=I \in \operatorname{Pos}_{k}$ :

Caso 1: $\lim _{n \rightarrow \infty} \frac{1}{n} d\left(I, Q_{n}(\omega)\right)=0$ para quase todo $\omega \in \Omega$.

Caso 2: Para quase todo $\omega \in \Omega$, existe uma geodésica $P^{2 t} \operatorname{com} P(\omega) \in \operatorname{Pos}_{k}$ tal que:

$$
\lim _{n \rightarrow \infty} \frac{1}{n} d\left(P^{2 n}, Q_{n}(\omega)\right)=0 .
$$

Onde, em ambos casos acima, $Q_{n}$ é a órbita de $I$ pelo cociclo $U$. Essencialmente, o Caso 1 é um caso particular da equação acima com $P=I$, por isso não há restrição demonstrar "apenas" para o Caso 2. Denotamos $d_{n}:=d\left(P^{2 n}, Q_{n}(\omega)\right)$, a equação (5.5) fica:

$$
\lim _{n \rightarrow \infty} \frac{1}{n} d_{n}=0
$$

Sejam $\mu_{1}>\ldots>\mu_{l}>0$ os autovalores distintos de $P$ (que também dependem continuamente de $P$ ) e definimos $\lambda_{i}:=\log \mu_{i}$. Sejam $W_{0}, \ldots, W_{l}$ os autoespaços associados ao $\mu_{1}, \ldots, \mu_{l}$ respectivamente. Até agora apenas sabemos (do Teorema K-M) que os $\lambda_{i}$ são funções mensuráveis de $\omega$. A partir daqui provaremos que os $\lambda_{i}$ 's são constantes em quase todo ponto:

Seja $\omega$ tal que existem as matrizes positivas $P$ e $Q$ satisfazendo:

$$
\left\{\begin{array}{l}
\lim _{n \rightarrow \infty} \frac{1}{n} d\left(P^{2 n}, Q_{n}(\omega)\right)=0 \\
\lim _{n \rightarrow \infty} \frac{1}{n} d\left(Q^{2 n}, Q_{n}(\omega)\right)=0 .
\end{array}\right.
$$

Pela desigualdade triangular:

$$
\begin{aligned}
& d\left(P^{2 n},\left[M(1, \omega)^{*}\right] Q^{2 n}\right) \leq \\
& d\left(P^{2 n}, Q_{n}(\omega)\right)+d\left(Q_{n}(\omega), Q_{n+1}(\omega)\right)+d\left(Q_{n+1}(\omega),\left[M(1, \omega)^{*}\right] Q^{2 n}\right) .
\end{aligned}
$$

Por isometria, temos:

$$
\begin{aligned}
& d\left(Q_{n}(\omega), Q_{n+1}(\omega)\right)=d\left(I, Q_{1}\left(T^{n+1} \omega\right)\right) \\
& d\left(Q_{n+1}(\omega),\left[M(1, \omega)^{*}\right] Q^{2 n}\right)=d\left(Q_{n}(T \omega), Q^{2 n}\right) .
\end{aligned}
$$

Dividindo a equação (5.8) por $n$ e levando $n \rightarrow \infty$, pelas equações (5.7), (5.9), (5.10) temos:

$$
\lim _{n \rightarrow \infty} \frac{1}{n} d\left(P^{2 n},[M(1, \omega)] Q^{2 n}\right)=0 .
$$

Segue do Lema 5.7 que $P$ e $Q$ são conjugadas. Portanto $\lambda_{i}(\omega)=\lambda_{i}(T \omega)$ num subconjunto de medida total de $\Omega$. Por ergodicidade de $T$, temos que $\lambda_{i}$ é 
constante $\mu$-qtp em $\Omega$.

Agora vamos provar a segunda parte do teorema. Definimos $V_{i}:=$ $W_{i} \oplus \cdots \oplus W_{l}$ e $V_{l+1}=\{0\}$. Dado $v \in \mathbb{R}^{k}$ com $v \in V_{i_{0}} \backslash V_{i_{0}+1}$, podemos decompor $v$ em:

$$
v=v_{i_{0}}+\sum_{i>i_{0}} v_{i}
$$

onde $i_{0}$ é tal que $v_{i_{0}} \neq 0$ e $v_{i} \in W_{i}$ para cada $i \in\left\{i_{0}, \ldots, l\right\}$. Para obter a igualdade (5.2), provaremos a seguinte afirmação:

Afirmação 5.8. $\lim _{n \rightarrow \infty} \frac{1}{n} \log \|M(n, \omega) v\|=\lim _{n \rightarrow \infty} \frac{1}{n} \log \left\|P^{n} v\right\|$ para todo $v \in \mathbb{R}^{k}$.

Prova da Afirmação 5.8. Usando a Proposição 5.5, temos:

$$
e^{-d_{n}} P^{2 n} \leq Q_{n} \leq e^{d_{n}} P^{2 n}
$$

Mas $Q_{n}=\left[M(n, \omega)^{*}\right](I)$, as desigualdades acima implicam:

$$
\begin{aligned}
& e^{-d_{n}}\left\langle P^{2 n} v, v\right\rangle \leq\left\langle\left[M(n, \omega)^{*}\right](I) v, v\right\rangle \leq e^{d_{n}}\left\langle P^{2 n} v, v\right\rangle \\
\Longrightarrow & e^{-d_{n}}\left\langle\left(P^{*}\right)^{n} P^{n} v, v\right\rangle \leq\left\langle M(n, \omega)^{*} M(n, \omega) v, v\right\rangle \leq e^{d_{n}}\left\langle\left(P^{*}\right)^{n} P^{n} v, v\right\rangle \\
\Longrightarrow & e^{-d_{n}}\left\langle P^{n} v, P^{n} v\right\rangle \leq\langle M(n, \omega) v, M(n, \omega) v\rangle \leq e^{d_{n}}\left\langle P^{n} v, P^{n} v\right\rangle \\
\Longrightarrow & e^{-\frac{d_{n}}{2}}\left\|P^{n} v\right\| \leq\|M(n, \omega) v\| \leq e^{\frac{d_{n}}{2}}\left\|P^{n} v\right\| .
\end{aligned}
$$

Tomando logaritmo nas desigualdades acima, obtemos:

$$
-\frac{d_{n}}{2}+\log \left\|P^{n} v\right\| \leq \log \|M(n, \omega) v\| \leq \frac{d_{n}}{2}+\log \left\|P^{n} v\right\| .
$$

Dividindo as desigualdades acima por $n$ e fazendo $n \rightarrow \infty$, temos pela equação (5.6):

$$
\lim _{n \rightarrow \infty} \frac{1}{n} \log \left\|P^{n} v\right\|=\lim _{n \rightarrow \infty} \frac{1}{n} \log \|M(n, \omega) v\|
$$

Voltamos à prova do Teorema de Oseledets. Usando a Afirmação 5.8, temos:

$$
\begin{aligned}
\lim _{n \rightarrow \infty} \frac{1}{n} \log \frac{\|M(n, \omega) v\|}{\|v\|} & =\lim _{n \rightarrow \infty} \frac{1}{n} \log \frac{\left\|P^{n}\left(v_{i_{0}}+\sum_{i>i_{0}} v_{i}\right)\right\|}{\|v\|} \\
& =\lim _{n \rightarrow \infty} \frac{1}{n} \log \frac{\left\|\mu_{i_{0}}^{n} v_{i_{0}}+\sum_{i>i_{0}} \mu_{i}^{n} v_{i}\right\|}{\|v\|} \\
& =\lambda_{i_{0}}+\lim _{n \rightarrow \infty} \frac{1}{n} \log \frac{\left\|v_{i_{0}}+\sum_{i>i_{0}}\left(\frac{\mu_{i}}{\mu_{i_{0}}}\right)^{n} v_{i}\right\|}{\|v\|} \\
& =\lambda_{i_{0}} .
\end{aligned}
$$


Na última linha, usamos $\lim _{n \rightarrow \infty}\left(\frac{\mu_{i}}{\mu_{i_{0}}}\right)^{n}=0$. Portanto provamos que para quase todo $\omega \in \Omega$, existem $\lambda_{1}(\omega)>\ldots>\lambda_{l}(\omega)$ e subespaços $\mathbb{R}^{k}=V_{1}(\omega) \supset \ldots \supset$ $V_{l}(\omega) \supset V_{l+1}(\omega)=\{0\}$ satisfazendo a equação (5.2).

Finalmente, $\lambda_{i}(T \omega)=\lambda_{i}(\omega)$ segue do Corolário 2.12 e Lema 5.7. Isso conclui a prova do teorema.

Outra consequência da demonstração do Teorema de Oseledets é que o drift $A$ do cociclo $U$ é a "média" dos exponentes de Lyapunov no seguinte sentido:

Proposição 5.9. Sejam $M: \mathbb{N} \times \Omega \rightarrow G L_{k}(\mathbb{R})$ cociclo à esquerda e $U$ definida por $U(n, \omega) P:=\left[M(n, \omega)^{*}\right] P$ ao cociclo associado a $M$. Então temos a seguinte relação:

$$
A=2\left[\sum_{i=1}^{l}\left(\operatorname{dim} V_{i}-\operatorname{dim} V_{i+1}\right) \lambda_{i}^{2}\right]^{\frac{1}{2}}
$$

onde $A$ é o drift de $U, \lambda_{1}, \ldots, \lambda_{l}$ são os exponentes de Lyapunov associados a $M$ e $V_{1}, \ldots, V_{l+1}$ é a bandeira de subespaços como no Teorema de Oseledets.

Prova. Sejam $\nu_{i}$ 's são os autovalores da matriz $P$ contando com multiplicidades. A equação (5.5) e a fórmula da distância (5.3) implicam que:

$$
\begin{aligned}
A & =\lim _{n \rightarrow \infty} \frac{1}{n} d\left(P^{2 n}, I\right)=\lim _{n \rightarrow \infty} \frac{1}{n}\left[\sum_{i=1}^{k}\left(\log \nu_{i}^{2 n}\right)^{2}\right]^{\frac{1}{2}} \\
& =\lim _{n \rightarrow \infty} \frac{1}{n}\left[4 n^{2} \sum_{i=1}^{k}\left(\log \nu_{i}\right)^{2}\right]^{\frac{1}{2}} \\
& =2\left[\sum_{i=1}^{l}\left(\operatorname{dim} V_{i}-\operatorname{dim} V_{i+1}\right) \lambda_{i}^{2}\right]^{\frac{1}{2}}
\end{aligned}
$$

que é a fórmula desejada.

\subsection{Exemplo de aplicação do Teorema de Oseledets}

No Capítulo 4 estudamos um cociclo das transformações de Möbius no disco hiperbólico. Agora iremos mostrar uma nova interpretação usando o Teorema de Oseledets. Ao mesmo tempo, veremos que neste exemplo os expoentes de Lyapunov serão dois valores distintos, e uma consequência é que 
o drift $A$ do cociclo no disco é positivo para todo $l \in\left(0, \frac{1}{2}\right)$ generalizando a Proposição 4.4. Primeiro, formalizaremos a interpretação:

Definimos o conjunto:

$$
S \operatorname{Pos}_{2}:=\left\{P \in \mathrm{Pos}_{2} \mid \operatorname{det} P=1\right\}
$$

$\mathrm{SPos}_{2}$ é uma subvariedade totalmente geodésica de $\mathrm{Pos}_{2}$. De fato, dados matrizes quaisquer $P, Q \in S \mathrm{Pos}_{2}$, o segmento geodésico definido por $\left[P^{\frac{1}{2}}\right]\left(P^{-\frac{1}{2}} Q P^{-\frac{1}{2}}\right)^{t}$ para $t \in[0,1]$ conecta as matrizes e além disso está contido em $\mathrm{SPOs}_{2}$.

A transformação de Möbius $\mathcal{T}$ associada a matriz

$$
\frac{1}{\sqrt{2}}\left(\begin{array}{cc}
i & 1 \\
-1 & -i
\end{array}\right)
$$

leva o disco $\mathbb{D}$ no semi-plano superior $\mathbb{H}:=\{x+i y \in \mathbb{C} \mid x, y \in \mathbb{R}, y>0\}$. Além disso, $\mathcal{T}$ é difeomorfismo e conforme, portanto induz uma métrica no $\mathbb{H}$ de modo que $\mathcal{T}$ é isometria.

Sejam $J_{1}, J_{2}: \mathbb{H} \rightarrow \mathbb{H}$ dadas por $J_{1}:=\mathcal{T} I_{1} \mathcal{T}^{-1}$ e $J_{2}:=\mathcal{T} I_{2} \mathcal{T}^{-1}$. Uma conta simples mostra que as isometrias $J_{1}, J_{2}$ são associadas aos matrizes:

$$
\tilde{M}_{1}=R D R^{-1} \text { e } \tilde{M}_{2}=D
$$

onde $D$ é matriz diagonal e $R$ é matriz "rotação anti-horária de 45 graus" descritas abaixo:

$$
D=\frac{1}{1-l^{2}}\left(\begin{array}{cc}
(1-l)^{2} & 0 \\
0 & (1+l)^{2}
\end{array}\right), \quad R=\frac{1}{\sqrt{2}}\left(\begin{array}{cc}
1 & -1 \\
1 & 1
\end{array}\right)
$$

Note que as matrizes $\tilde{M}_{1}$ e $\tilde{M}_{2}$ estão em $\mathrm{SPos}_{2}$. Os seguintes vetores unitários formam uma base de $T_{I} \mathrm{Pos}_{2}$ :

$$
e_{1}:=\frac{1}{\sqrt{2}}\left(\begin{array}{ll}
0 & 1 \\
1 & 0
\end{array}\right), \quad \text { e } \quad e_{2}:=\frac{1}{\sqrt{2}}\left(\begin{array}{cc}
1 & 0 \\
0 & -1
\end{array}\right) .
$$

Definimos as geodésicas de velocidade unitárias $\tilde{\alpha}(t):=e^{t e_{1}}$ e $\tilde{\beta}(t):=e^{t e_{2}}$. Uma conta direta mostra que $J_{1}$ preserva a geodésica $\tilde{\alpha}$ transladando-o por uma distância de $4 \sqrt{2} \tanh ^{-1} l$. Analogamente, $J_{2}$ preserva a geodésica $\tilde{\beta}$ transladando-o por uma distância de $4 \sqrt{2} \tanh ^{-1} l$. Definimos também $\tilde{M}_{3}:=$ $\tilde{M}_{1}^{-1}$ e $\tilde{M}_{4}:=\tilde{M}_{2}^{-1}$. 
Definimos o cociclo à esquerda $\tilde{M}: \mathbb{N} \times\{1,2,3,4\}^{\mathbb{N}} \rightarrow S L_{2}(\mathbb{R})^{\dagger}$ por:

$$
\left\{\begin{array}{l}
\tilde{M}(0, \omega)=I \\
\tilde{M}(n, \omega)=\tilde{M}_{\omega_{n-1}} \cdots \tilde{M}_{\omega_{0}}, \quad n \geq 1
\end{array}\right.
$$

A ação de $G L_{2}(\mathbb{R})$ sobre $\operatorname{Pos}_{2}(\mathbb{R})$ induz naturalmente uma ação de $S L_{2}(\mathbb{R})$ sobre o $\operatorname{SPos}_{2}(\mathbb{R})$. Como consequência do Teorema de K-M, para quase todo $\omega \in \Omega$ existe uma matriz $P(\omega) \in \operatorname{SPos}_{2}(\mathbb{R})$ tal que $\operatorname{det} P=e^{\lambda_{1}} e^{\lambda_{2}}$ ( $\lambda_{1}$ e $\lambda_{2}$ são os expoentes de Lyapunov), donde obtemos $\lambda_{1}+\lambda_{2}=0$.

Os dois fatos anteriores mostram que os expoentes de Lyapunov do cociclo à esquerda $\tilde{M}$ são $\{\lambda,-\lambda\} \operatorname{com} \lambda \neq 0$. Sejam $\tilde{U}$ o cociclo associado ao $\tilde{M}$ e $\tilde{A}$ o drift de $\tilde{U}$, pela Proposição 5.9 temos:

$$
\tilde{A}=2\left(\lambda^{2}+\lambda^{2}\right)^{\frac{1}{2}}=2 \sqrt{2} \lambda .
$$

Em particular, temos $\tilde{A}>0$.

Agora, seja $\tilde{g}$ a métrica traço definida na Seção 5.2. Iremos verificar que $\left(S \operatorname{Pos}_{2}, \tilde{g}\right)$ e $(\mathbb{D}, g)$ são "isométricas a menos de um múltiplo" no seguinte sentido:

Proposição 5.10. Definimos uma nova métrica $g_{2}$ em $\mathbb{D}$ :

$$
g_{2, p}(u, v):=\frac{2\langle u, v\rangle}{\left(1-|z|^{2}\right)^{2}}
$$

Dados $p \in \mathbb{D}, P \in S$ Pos $_{2}, v \in T_{p} \mathbb{D}$ e $V \in T_{P} S P_{o s}$. Existe uma isometria $F: \mathbb{D} \rightarrow S$ Pos $_{2}, \operatorname{com} F(p)=P, d F_{p}(v)=d F_{P}(V)$.

Prova. Uma conta direta mostra que curvatura de $\left(\mathbb{D}, g_{2}\right)$ é -2 e sabemos que $\mathbb{D}$ é simplesmente conexo.

Como $\mathrm{SPos}_{2}$ é uma subvariedade totalmente geodésica de $\mathrm{Pos}_{2}$, a conexão Riemanniana em $\mathrm{SPos}_{2}$ é a mesma de $\mathrm{Pos}_{2}$, em particular os tensores de curvatura são iguais. A curvatura ${ }^{\ddagger}$ de $S P_{o s}$ em $I$ é dada por (cf. [Lang, p.338, Teo.3.9]):

$$
\frac{\left\langle R\left(e_{1}, e_{2}\right) e_{1}, e_{2}\right\rangle_{I}}{\left\|e_{1} \wedge e_{2}\right\|}=\frac{\left\langle\left[\left[e_{1}, e_{2}\right], e_{1}\right], e_{2}\right\rangle_{I}}{\left\|e_{1} \wedge e_{2}\right\|}=-2 .
$$

Além disso, como o grupo de isometrias é transitivo sobre $\mathrm{SPos}_{2}$, este tem a curvatura constante. Um teorema clássico de geometria Riemanniana (cf. [do Carmo, p.163]) mostra que $S \mathrm{Pos}_{2}$ é isométrica a (D,$\left.g_{2}\right)$.

\footnotetext{
${ }^{\dagger} S L_{2}(\mathbb{R}):=\left\{M \in G L_{2}(\mathbb{R}) \mid \operatorname{det} M=1\right\}$

${ }^{\ddagger}$ As definições da curvatura em [Lang] e [do Carmo] diferem por um sinal.
} 
Como os vetores $e_{1}$ e $e_{2}$ são ortogonais, na Proposição 5.10 podemos fazer $F(0):=I, d F_{0}((1,0))=e_{1}$ e $d F_{0}((0,1))=e_{2}$. Com essa definição temos: $F\left(\alpha\left(\frac{t}{\sqrt{2}}\right)\right)=\tilde{\alpha}(t)$ e $F\left(\beta\left(\frac{t}{\sqrt{2}}\right)\right)=\tilde{\beta}(t)$. Seja $u$ o cociclo sobre $\mathbb{D}$ definido no Capítulo 4 , o drift de $u$ na nova métrica $g_{2}$ passa a ser: $A_{2}=\sqrt{2} A$, onde $A$ é o drift de $u$ na métrica $g$.

Como a distância transladada de $J_{1}$ é $4 \sqrt{2} \tanh ^{-1} l$ que é dois vezes $2 \sqrt{2} \tanh ^{-1} l$, que é a distância transladada de $I_{1}$ na métrica $g_{2}$, temos $\tilde{A}=2 A_{2}=2 \sqrt{2} A$. Pela equação (5.11) concluímos que:

Proposição 5.11. Seja u o cociclo definido como no Capítulo 4 e seja $A$ o seu drift. Então:

$$
A=\lambda \text {. }
$$

Vimos no Capítulo 4 que $A>0$ quando $l>\sqrt{2}-1$, portanto vale $\lambda>0$ nesse caso. De fato vale $\lambda>0$ para todo $l>0$, como consequência do Teorema de Furstenberg§ ${ }^{\S}$ Cujo o enunciado é:

Teorema 5.12. Consideremos $\Omega=\{1, \ldots, l\}^{\mathbb{N}}, \mu$ a medida de Bernoulli com pesos $p_{1}, \ldots, p_{l}$ positivos e $\sum p_{i}=1$. Sejam $M_{1}, \ldots, M_{l} \in S L(2, \mathbb{R})$ fixados.

Seja $M: \mathbb{N} \times \Omega \rightarrow S L_{2}(\mathbb{R})$ um cociclo à esquerda definido por:

$$
M(n, \omega):=M_{\omega_{n-1}} \cdots M_{\omega_{0}}
$$

Supondo que:

1. O grupo gerado por $\left\{M_{1}, \ldots, M_{l}\right\}$ é não-compacto.

2. Não existe um conjunto $L \subset \mathbb{P}^{1}$, com número de elementos $\# L$ igual a 1 ou 2 tal que $M_{i}(L)=L$ para todo $i \in\{1, \ldots, l\}$.

Então o exponente de Lyapunov superior de $M$ é positivo.

No nosso caso, definimos $\tilde{G}:=$ Grupo gerado por $\tilde{M}_{1}$ e $\tilde{M}_{2}$. Logo:

1. $\tilde{G}$ é não-compacto, pois $\left\{\tilde{M}_{2}^{n}\right\}_{n \in \mathbb{N}}$ é uma sequência ilimitada em $G_{\mu}$.

2. Para cada $i \in\{1,2\}$, seja $L_{i}$ tal que $\tilde{M}_{i}\left(L_{i}\right)=L_{i}$. Então $L_{1} \subset$ $\{\mathbb{R}(1,1), \mathbb{R}(1,-1)\}$ e $L_{2} \subset\{\mathbb{R}(1,0), \mathbb{R}(0,1)\}$. Logo $L_{1} \cap L_{2}=\emptyset$, isto é: nenhuma direção é fixada pelas ambas matrizes ao mesmo tempo.

Pelo Teorema de Furstenberg aplicado ao $\tilde{M}$, obtemos que $\lambda>0$. Isso generaliza o resultado da Proposição 4.4, que provamos no capítulo anterior para o caso $l>\sqrt{2}-1$.

${ }^{\S}$ Cf. [Furstenberg, 1963] 


\section{Preliminares Geométricos}

Este capítulo é dividido em duas seções de tópicos independententes que servem de auxílio para outros capítulos. A primeira é um Lema que usaremos na demonstração do Teorema de K-M. A segunda é dedicada a verificar que uma variedade Riemanniana de curvatura não-positiva, completa e simplesmente conexa é uniformemente convexa e satisfaz a condição de curvatura não-positiva de Busemann.

\subsection{Um Lema Geométrico}

O seguinte lema diz que, em um espaço uniformemente convexo, se um triângulo com vértices $x, y$ e $z$ é tal que:

$$
d(y, x)+d(x, z) \simeq d(y, z)
$$

(a desigualdade triangular está perto de ser uma igualdade), então o vértice $x$ está próximo do segmento $\overline{y z}$ (cf. Fig. 6.1).

Lema 6.1. Seja $(Y, d)$ um espaço métrico uniformemente convexo. Sejam $x, y, z \in Y$, supondo que $d(y, z) \geq d(y, x)$ e assumimos que existe $\delta \in[0,1]$ tal que:

$$
d(y, x)+d(x, z) \leq d(y, z)+\delta d(x, y)
$$

Seja $\tilde{x}$ o ponto sobre a geodésica $\overline{y z}$ tal que $d(y, \tilde{x})=d(y, x)$. Então existe uma função $h:[0,1] \rightarrow[0,2]$ (que não depende de $x$ e y) com $\lim _{s \rightarrow 0} h(s)=0$ tal que:

$$
d(x, \tilde{x}) \leq h(\delta) d(y, x) .
$$

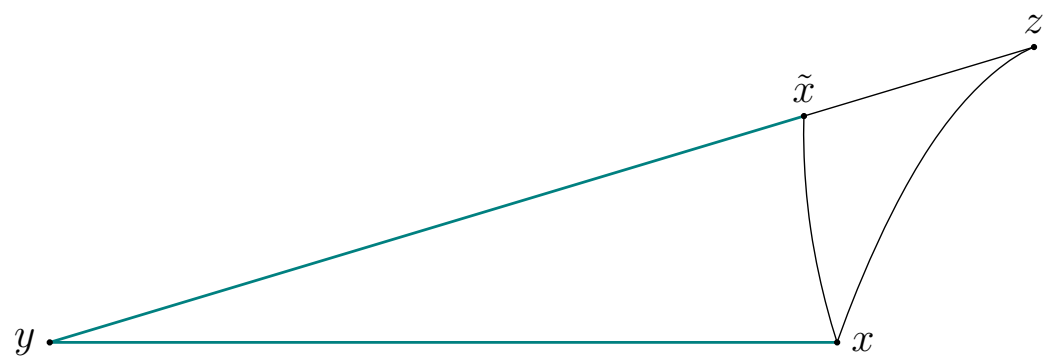

Figura 6.1: Observe que o segmento geodésico de $x$ ao $\tilde{x}$ é pequeno. 
Prova. Seja $w$ ponto médio entre $x$ e $\tilde{x}$. Pela convexidade uniforme:

$$
d(w, z) \leq \max \{d(x, z), d(\tilde{x}, z)\}=d(x, z) .
$$

Por hipótese do lema, $d(x, z) \leq d(y, z)-d(y, x)+\delta d(x, y)$ e usando a desigualdade acima temos:

$$
d(w, z) \leq d(y, z)-(1-\delta) d(x, y)
$$

Por definição, $d(\tilde{x}, z)=d(y, z)-d(y, x)$, a desigualdade acima e a desigualdade triangular implicam:

$$
d(y, x)(1-\delta) \leq d(y, z)-d(w, z) \leq d(y, w)
$$

Usando a Definição 2.3 de convexidade uniforme, com $R$ := $\max \{d(y, x), d(y, \tilde{x})\}=d(y, x)$, da desigualdade obtemos:

$$
\begin{aligned}
& 1-\delta \leq \frac{d(y, w)}{R} \leq g\left(\frac{d(x, \tilde{x})}{2 R}\right) \\
\Longrightarrow & g^{-1}(1-\delta) \geq \frac{d(x, \tilde{x})}{2 R} \\
\Longrightarrow & 2 g^{-1}(1-\delta) d(y, x)=2 g^{-1}(1-\delta) R \geq d(x, \tilde{x}) .
\end{aligned}
$$

Definimos $h(s):=2 g^{-1}(1-s)$, segue da definição de $g$ que $h$ é crescente e $\lim _{s \rightarrow 0} h(s)=0$.

\subsection{Variedades de Curvatura Seccional Não-positiva}

Ao longo da seção consideremos $Y$ uma variedade Riemanniana completa, simplesmente conexa e com curvaturas seccionais não-positivas.

Proposição 6.2. A variedade $Y$ é uniformemente convexa e possui a curvatura não-positiva no sentido de Busemann.

Observação. A Proposição 6.2 pode ser generalizada para os espaços CAT(0). A ideia da demonstração de convexidade uniforme para os espaços CAT(0) pode ser encontrada na prova dessa proposição; A propriedade de Busemann NPC segue imediatamente da definição de CAT(0).

Para mostrar a proposição 6.2 acima usaremos Teorema de Hadamard, Campo de Jacobi e uma consequência do Teorema de Comparação de Rauch (cf. [do Carmo, p.218]), os quais enunciaremos abaixo: 
Teorema 6.3 (Hadamard). Para todo $y \in Y$, a aplicação $\exp _{y}: T_{y} Y \rightarrow Y$ é difeomorfismo.

Lembramos que um campo vetorial $J$ ao longo de uma geodésica $\gamma$ : $[0, b] \rightarrow Y$ é dito campo de Jacobi se este satisfaz a equação de Jacobi:

$$
J^{\prime \prime}+R\left(\gamma^{\prime}, J\right) \gamma^{\prime}=0
$$

onde $R$ é o tensor curvatura (cf. [do Carmo, p.89]).

E por último, o corolário que segue do Teorema de Hadamard e Rauch é o seguinte:

Corolário 6.4 (do Teorema de Rauch $^{a}$ ). Sejam $Y_{1}$ e $Y_{2}$ variedades Riemannianas completas, simplesmente conexas com curvaturas seccionais $K_{1}$ e $K_{2}$ tais que:

$$
K_{1} \leq K_{2} \leq 0
$$

Consideremos os triângulos $\triangle x_{1} y_{1} z_{1}$ em $Y_{1}$ e $\triangle x_{2} y_{2} z_{2}$ em $Y_{2}$ tais que $d\left(z_{1}, x_{1}\right)=d\left(z_{2}, x_{2}\right), d\left(z_{1}, y_{1}\right)=d\left(z_{2}, y_{2}\right)$ e $\angle x_{1} z_{1} y_{1}=\angle x_{2} z_{2} y_{2}$. Então:

$$
d\left(x_{1}, y_{1}\right) \geq d\left(x_{2}, y_{2}\right)
$$

${ }^{a} \mathrm{O}$ Teorema de Toponogov generaliza este corolário

Agora começamos a prova da Proposição 6.2.

Prova de convexidade uniforme. Sejam $x, y, z$ vértices de um triângulo em $Y$. Construímos os triângulos Euclidianos de vértices $\tilde{z}, \tilde{m}, \tilde{x}$ e $\tilde{z}, \tilde{m}, \tilde{y}$ respectivamente, de modo que:

$$
\left\{\begin{array}{l}
d(\tilde{z}, \tilde{m})=d\left(z, m_{x y}\right), \\
\angle \tilde{z} \tilde{m} \tilde{x}=\angle z m_{x y} x, \text { e } \angle \tilde{z} \tilde{m} \tilde{y}=\angle z m_{x y} y, \\
d(\tilde{y}, \tilde{m})=d(\tilde{x}, \tilde{m})=d\left(x, m_{x y}\right) .
\end{array}\right.
$$

Pelo Corolário 6.4 temos

$$
\tilde{R}:=\max \{d(\tilde{z}, \tilde{x}), d(\tilde{z}, \tilde{y})\} \leq \max \{d(z, x), d(z, y)\}=: R .
$$

Portanto pela equação (2.2) temos:

$$
\begin{aligned}
\left(d\left(z, m_{x y}\right)\right)^{2}=(d(\tilde{z}, \tilde{m}))^{2} & \leq \tilde{R}^{2}-(d(\tilde{x}, \tilde{m}))^{2} \\
& \leq R^{2}-\left(d\left(x, m_{x y}\right)\right)^{2} .
\end{aligned}
$$


Lembrando que $d(z, x) \leq R$ e $d\left(x, m_{x y}\right)=\frac{d(x, y)}{2}$, dividimos a desigualdade acima por $R^{2}$, segue que:

$$
\frac{d\left(z, m_{x y}\right)}{R} \leq g\left(\frac{d(x, y)}{2 R}\right)
$$

$\operatorname{para} g(t):=\left(1-t^{2}\right)^{\frac{1}{2}}$

Prova de curvatura não-positiva. Sejam $x, y, z \in Y$ três pontos nãocolineares. Consideremos a variação de geodésicas $f:[0,1] \times[0,1] \rightarrow Y$ de modo que para cada $s \in[0,1]$ fixo, a curva $\gamma_{s}(t):=f(s, t)$ é geodésica com $\gamma_{0}$ de $z$ ao $x$ e $\gamma_{1}$ de $z$ ao $y$. Além disso, também colocamos a condição $f(s, 0)=z$ para todo $s \in[0,1]$ e $f(s, 1)$ é a geodésica de $x$ ao $y$.

Segue da definição que $J_{s}(t):=\frac{\partial f}{\partial s}(s, t)$ é um campo de Jacobi ao longo da geodésica $\gamma_{s}$ com $J_{s}(0)=0$. A não-colinearidade implica $J_{s}^{\prime}(0) \neq 0$ para todo $s \in[0,1]$. Notemos que $f\left(s, \frac{1}{2}\right)$ é uma curva de $m_{x z}$ ao $m_{y z}$, logo condição $\frac{\partial^{2}}{\partial t^{2}}\left\|J_{s}(t)\right\| \geq 0$ implica:

$$
d\left(m_{x z}, m_{y z}\right) \leq \int_{0}^{1}\left\|J_{s}\left(\frac{1}{2}\right)\right\| d s \leq \frac{1}{2} \int_{0}^{1}\left\|J_{s}(1)\right\| d s=\frac{1}{2} d(x, y)
$$

que é a condição de não-positividade de Busemann. Portanto é suficiente que $\frac{\partial^{2}}{\partial t^{2}}\left\|J_{s}(t)\right\| \geq 0$. Como a prova é análoga para todo $s \in[0,1]$, faremos essa verificação apenas para $J(t):=J_{0}(t)$ :

$$
\begin{aligned}
\frac{\partial^{2}}{\partial t^{2}}\|J\| & =\frac{\partial}{\partial t}\left(\langle J, J\rangle^{-\frac{1}{2}}\left\langle J^{\prime}, J\right\rangle\right) \\
& =\langle J, J\rangle^{-\frac{3}{2}}\left(\left\|J^{\prime}\right\|^{2}\|J\|^{2}-\left\langle J^{\prime}, J\right\rangle^{2}+\left\langle J^{\prime \prime}, J\right\rangle\|J\|^{2}\right) \\
& \geq\langle J, J\rangle^{-\frac{3}{2}}\left(0-\left\langle R\left(\gamma_{0}^{\prime}, J\right) \gamma_{0}^{\prime}, J\right\rangle\|J\|^{2}\right)
\end{aligned}
$$

onde na última desigualdade usamos a Desigualdade de Cauchy-Schwarz e a Equação de Jacobi (6.1). Como a curvatura seccional é não-positiva então $\left\langle R\left(\gamma_{0}^{\prime}, J\right) \gamma_{0}^{\prime}, J\right\rangle \leq 0$ e portanto a desigualdade acima implica: $\frac{\partial^{2}}{\partial t^{2}}\|J\| \geq 0$. 


\section{Propriedades de Cociclos Subaditivos}

Este é um capítulo preparatório para a demonstração do Teorema K-M. Vamos provar o "Lema de Pliss Ergódico", devido a K-M, que é o ingrediente mais importante na prova do Teorema K-M e possui outras aplicações interessantes [Karlsson e Ledrappier, 2008]. Na segunda metade do capítulo, provaremos o Teorema de Kingman.

Este capítulo é a parte mais técnica da demonstração do Teorema de K-M, dependendo da preferência do leitor, pode-se optar por ler a prova do Teorema K-M no Capítulo 8 primeiro. Também referimos a tabela da página 11, para auxiliar na compreensão da relação entre os resultados que apresentados ao longo do texto.

\subsection{Lema de Pliss Ergódico e algumas propriedades}

A proposição abaixo, obtida por K-M, é uma versão ergódica do Lema de Pliss (cf. [Mañé, p.356]) sobre sequências:

Proposição 7.1 (Lema de Pliss Ergódico). Seja $T: \Omega \rightarrow \Omega$ ergódica e $a_{n}$ um cociclo subaditivo no sistema dinâmico $(\Omega, \mathcal{A}, \mu ; T)$ satisfazendo:

$$
\int_{\Omega} a^{+}(1, \omega) d \mu<\infty .^{a}
$$

Defina:

$$
A:=\lim _{n \rightarrow \infty} \frac{1}{n} \int_{\Omega} a(n, \omega) d \mu .
$$

Se $A>-\infty$, então para quase todo $\omega \in \Omega$, e para todo $\epsilon>0$ existem $K(\omega, \epsilon) \in \mathbb{N}$ e uma sequência crescente $\left\{n_{i}\right\}_{i \in \mathbb{N}} \rightarrow \infty$ tais que:

$$
a\left(n_{i}, \omega\right)-a\left(n_{i}-k, T^{k} \omega\right) \geq(A-\epsilon) k \quad \forall k \in \mathbb{N} \text { tal que } K \leq k \leq n_{i}
$$

\footnotetext{
${ }^{a}$ Definimos $a^{+}(1, \omega):=\max \left(a_{1}(\omega), 0\right)$
}

Em outras palavras, seja:

$E_{\epsilon}:=\left\{\begin{array}{l|l}\omega \in \Omega & \begin{array}{c}\text { existem } K(\omega) \text { e infinitos } n \text { tais que, para todo } k, K \leq k \leq n \\ a\left(n_{i}, \omega\right)-a\left(n_{i}-k, T^{k} \omega\right) \geq(A-\epsilon) k\end{array}\end{array}\right\}$, 


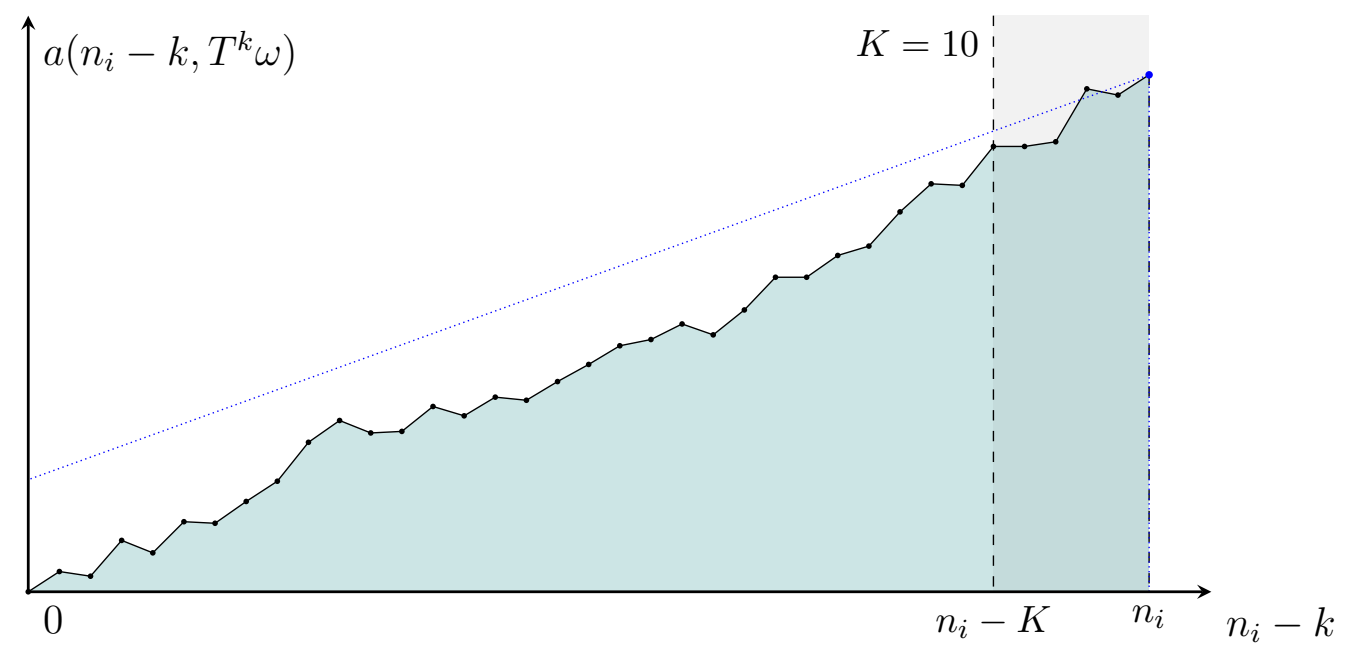

Figura 7.1: Observe que a reta de inclinação $(A-\epsilon)$ passando por ponto $\left(n_{i}, a\left(n_{i}-\right.\right.$ $\left.k, T^{k} \omega\right)$ ), atinge o "horizonte" dada pela reta vertical $n_{i}-k=0$, se os últimos $K=10$ pontos fossem ignorados.

e seja:

$$
E:=\bigcap_{\epsilon>0} E_{\epsilon}
$$

Então $\mu(E)=1$.

O significado do Lema de Pliss pode ser melhor entendido observando a Figura 7.1. Fixado $\epsilon$, existe um $K \in \mathbb{N}$ satisfazendo as seguintes propriedades. Para cada $n_{i}$ olhamos o gráfico de $a\left(n_{i}-k, T^{k} \omega\right)$ como uma função de $n_{i}-k$. Notemos que se $n_{i}$ satisfaz a equação (7.1) então o respectivo gráfico associado está abaixo da reta de inclinação $(A-\epsilon)$ passando pelo ponto $\left(n_{i}, a\left(n_{i}, \omega\right)\right)$, com a exceção talvez dos últimos $K$ pontos (i.e. $\left.\left\{n_{i}-(K-1), n_{i}-(K-2), \ldots, n_{i}\right\}\right)$.

Para mostrar o Lema de Pliss precisamos de diversas ferramentas. Começaremos mostrando:

Lema 7.2 (F.Riesz). Seja $c_{0}, c_{1}, \ldots, c_{n}$ uma sequência finita de números reais. Dizemos que $c_{u}$ é um líder ${ }^{a}$ se:

$$
\min \left\{c_{u}, c_{u}+c_{u+1}, \ldots, c_{u}+\ldots+c_{n}\right\} \leq 0
$$

Então a soma dos líderes é não-negativa, ou seja:

$$
\sum_{c_{u} \text { é líder }} c_{u} \leq 0
$$

(Se o conjunto de líderes é vazio, o somatório acima é 0).

${ }^{a}$ Alguns autores definem o líder satisfazendo a desigualdade com sinal trocado (i.e. $\geq 0$ ). Nesse caso, a conclusão do Lema de Riesz também difere por um sinal (i.e. $\geq 0$ ). 
Prova. Usamos indução sobre $n$. Para $n=0$ é trivial, pois o único elemento $c_{0}$ é líder se e somente se $c_{0} \leq 0$. Agora consideremos $n \geq 1$ e supondo que vale a desigualdade para todos os naturais menores que $n$, vamos mostrar que vale para $n$.

Caso 1: Se $c_{0}$ não é líder então todos os líderes são líderes da subsequência $c_{1}, \ldots, c_{n}$ de $n-1$ elementos, daí vale o resultado.

Caso 2: Se $c_{0}$ é líder, escolhemos $k$ o menor inteiro tal que:

$$
c_{0}+\ldots+c_{k} \leq 0
$$

Afirmamos que $c_{1}, \ldots, c_{k}$ são líderes também. Suponhamos, por absurdo, que algum desses $c_{i}$ não é líder, logo:

$$
c_{i}+\ldots+c_{k}>0
$$

daí por (7.2) e (7.3) temos:

$$
c_{0}+\cdots+c_{i-1} \leq-\left(c_{i}+\cdots+c_{k}\right)<0
$$

contradizendo a hipótese que $k$ é menor inteiro satisfazendo (7.2). Portanto temos

$$
\sum\left(\begin{array}{c}
\text { líderes da sequência } \\
\left\{c_{0}, \ldots, c_{n}\right\}
\end{array}\right)=\left(c_{0}+\ldots+c_{k}\right)+\sum\left(\begin{array}{c}
\text { líderes da sequência } \\
\left\{c_{k+1}, \ldots, c_{n}\right\}
\end{array}\right) .
$$

Como $\left(c_{0}+\ldots+c_{k}\right) \leq 0$ (por escolha de $\left.k\right)$ e $\sum\left(\begin{array}{c}\text { líderes da sequência } \\ \left\{c_{k+1}, \ldots, c_{n}\right\}\end{array}\right) \leq 0$ por hipótese de indução, pela desigualdade acima o lema está provado.

\subsection{Lema de Pliss Versão Fraca}

O lema que apresentaremos a seguir, em contraste com o Lema de Pliss, exigimos que $T$ seja apenas uma transformação preservando a medida, não precisamos da ergodicidade.

Lema 7.3. Seja $T: \Omega \rightarrow \Omega$ preservando a medida $\mu$, e sejam $a_{n}$ e $A$ definidas como na Proposição 7.1. Supondo que $A>0$, então o conjunto:

$$
\tilde{E}:=\left\{\begin{array}{l|l}
\omega \in \Omega & \begin{array}{c}
\exists \text { infinitos } n \text { tais que, } \forall k \text { com } 0 \leq k \leq n \\
a(n, \omega)-a\left(n-k, T^{k} \omega\right) \geq 0
\end{array}
\end{array}\right\}
$$

tem medida positiva, i.e. $\mu(\tilde{E})>0$. 
Prova. Usamos a notação $a_{n}:=\int_{\Omega} a(n, \omega) d \mu$ ao longo da demonstração. Para cada $i \in \mathbb{N}$, definimos o conjunto:

$W_{i}:=\left\{\omega \in \Omega \mid a(i, \omega)-a\left(i-k, T^{k} \omega\right)<0\right.$ para algum $k$ tal que $\left.0 \leq k \leq i\right\}$.

Segue da definição acima que:

$$
\tilde{E}=\left\{\omega \in \Omega \mid \text { existem infinitos } i \text { 's tais que } \omega \in W_{i}^{c}\right\}
$$

Definimos:

$$
B_{n}:=\left\{\omega \in \Omega \mid n \geq f_{n}(\omega)>\frac{A}{3 a_{1}^{+}} n\right\}
$$

onde $f_{n}=\sum_{j=0}^{n} \mathcal{X}_{W_{j}^{c}}$ e $a_{1}^{+}=\int_{\Omega} a^{+}(1, \omega) d \mu$, com $\mathcal{X}_{W_{j}^{c}}$ a função característica do conjunto $W_{j}^{c}$. Note que:

$B_{n} \subset C_{n}:=\left\{\omega \in \Omega \mid\right.$ a quantidade de $i$ 's tais que $\omega \in W_{i}^{c}$ é maior que $\left.\frac{A}{3 a_{1}^{+}} n i\right\}$, ainda temos $C_{n+1} \subset C_{n}$ para todo $n \in \mathbb{N}$ e:

$$
\tilde{E} \supset \bigcap_{n \geq 1} C_{n}
$$

Mostraremos existem $\delta>0$ e $N>0$ tais que $\mu\left(B_{n}\right) \geq \delta$ para todo $n>N$. Isso junto com a relação acima (7.4) garantem que $\mu(\tilde{E})>0$.

Afirmação 7.4. Existe $N>0$ tal que para todo $n>N$

$$
\int_{B_{n}} a^{+}(1, \omega) d \mu>\frac{A}{3}
$$

Prova da Afirmação 7.4. Observe que

$$
\begin{aligned}
\sum_{j=0}^{n} \int_{W_{j}^{c}} a^{+}(1, \omega) d \mu & =\int_{\Omega} f_{n}(\omega) a^{+}(1, \omega) d \mu \\
& =\int_{B_{n}} f_{n}(\omega) a^{+}(1, \omega) d \mu+\int_{B_{n}^{c}} f_{n}(\omega) a^{+}(1, \omega) d \mu \\
& \leq n \int_{B_{n}} a^{+}(1, \omega) d \mu+\frac{A}{3 a_{1}^{+}} n \int_{B_{n}^{c}} a^{+}(1, \omega) d \mu \\
& \leq n \int_{B_{n}} a^{+}(1, \omega) d \mu+\frac{A}{3} n
\end{aligned}
$$


Dividindo por $n$, temos:

$$
\int_{B_{n}} a^{+}(1, \omega) d \mu \geq \frac{1}{n} \sum_{j=0}^{n} \int_{W_{j}^{c}} a^{+}(1, \omega) d \mu-\frac{A}{3} .
$$

Para provar a afirmação, é suficiente mostrar que o primeiro termo à direita da desigualdade (7.5) satisfaz:

$$
\sum_{j=0}^{n} \int_{W_{j}^{c}} a^{+}(1, \omega) d \mu>\frac{2 A}{3} n
$$

Definimos:

$$
b_{i}(\omega):=a(i, \omega)-a(i-1, T \omega) .
$$

Usando a subaditividade temos:

$$
a^{+}(1, \omega) \geq a(1, \omega) \geq b_{i}(\omega)
$$

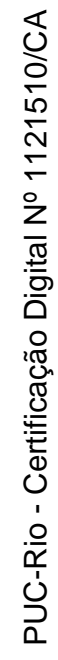

Daí:

$$
\sum_{i=1}^{n} \int_{W_{i}^{c}} a^{+}(1, \omega) d \mu \geq \sum_{i=1}^{n} \int_{W_{i}^{c}} b_{i}(\omega) d \mu .
$$

Por outro lado, a partir da definição temos:

$$
\begin{aligned}
a_{n}=\int_{\Omega} a(n, \omega) d \mu & =\sum_{i=0}^{n} \int_{\Omega} b_{i}(\omega) d \mu \\
& =\sum_{i=0}^{n}\left\{\int_{W_{i}} b_{i}(\omega) d \mu+\int_{W_{i}^{c}} b_{i}(\omega) d \mu\right\} \\
\Longrightarrow a_{n}-\sum_{i=0}^{n} \int_{W_{i}} b_{i}(\omega) d \mu & =\sum_{i=0}^{n} \int_{W_{i}^{c}} b_{i}(\omega) d \mu .
\end{aligned}
$$

Como $\lim _{n \rightarrow \infty} \frac{a_{n}}{n}=A>0$, existe $N$ tal que:

$$
a_{n}>\frac{2 A}{3} n, \quad \text { para todo } n \geq N
$$


Observe que o segundo termo à esquerda da equação (7.7) é igual a:

$$
\begin{aligned}
\sum_{i=0}^{n} \int_{W_{i}} b_{i}(\omega) d \mu & =\sum_{k=0}^{n} \int_{W_{n-k}} b_{n-k}(\omega) d \mu, \quad \text { onde } k:=n-i \\
& =\int_{\Omega} \sum_{\substack{k=0\\
}}^{n}\left(b_{n-k}(\omega) \mathcal{X}_{W_{n-k}}\right) d \mu \\
& =\int_{\Omega} \sum_{\substack{0 \leq k \leq n \\
T^{k} \omega \in W_{n-k}}} b_{n-k}\left(T^{k} \omega\right) d \mu
\end{aligned}
$$

Para cada $\omega \in \Omega$ e $k, 0 \leq k \leq n$, definimos a sequência $c_{k}:=b_{n-k}\left(T^{k} \omega\right)$. Observe que se $T^{k} \omega \in W_{n-k}$ então:

$$
\begin{aligned}
& b_{n-k}\left(T^{k} \omega\right)+\ldots+b_{n-j}\left(T^{j} \omega\right)=a\left(n-k, T^{k} \omega\right)-a\left(n-1-j, T^{j+1} \omega\right)<0, \\
& \text { para algum } j, \text { com } k \leq j \leq n . \\
\Longrightarrow & c_{k}+\ldots+c_{j}<0, \quad \text { para algum } j, \text { com } k \leq j \leq n . \\
\Longrightarrow & \min \left\{c_{k}, c_{k}+c_{k+1}, \ldots, c_{k}+\cdots+c_{n}\right\}<0,
\end{aligned}
$$

isso é, $c_{k}$ é líder. Aplicamos o Lema de Riesz 7.2 para a sequência $\left\{c_{u}\right\}$ :

$$
\sum_{\substack{0 \leq k \leq n \\ T^{k} \omega \in W_{n-k}}} b_{n-k}\left(T^{k} \omega\right)=\sum_{c_{k} \text { é líder }} c_{k} \leq 0
$$

A equação acima junto com (7.9) mostram que:

$$
\sum_{j=0}^{n} \int_{W_{i}} b_{i}(\omega) d \mu \leq 0
$$

Usando a desigualdade acima e (7.8) na equação (7.7), provamos:

$$
\sum_{i=0}^{n} \int_{W_{i}^{c}} b_{i}(\omega) d \mu>\frac{2 A}{3} n \quad \text { para todo } n>N
$$

Pelo argumento inicial vale (7.6), e portanto vale (7.5), isso conclui a prova da afirmação.

Afirmação 7.5. Existe $\delta>0$ tal que se $B \subset \Omega$ é um conjunto com $\mu(B)<\delta$ então:

$$
\int_{B} a^{+}(1, \omega) d \mu<\frac{A}{3} \text {. }
$$


Prova da Afrmação 7.5. Suponhamos por absurdo que existem conjuntos $S_{n}$ tais que $\lim _{n \rightarrow \infty} \mu\left(S_{n}\right)=0$ e:

$$
\int_{S_{n}} a^{+}(1, \omega) d \mu \geq \frac{A}{3}
$$

Mas $0 \leq \mathcal{X}_{S_{n}}(\omega) a^{+}(1, \omega) \leq a^{+}(1, \omega)$, e $\int_{\Omega} a^{+}(1, \omega) d \mu<\infty$, pelo Teorema da Convergência Dominada para $\mathcal{X}_{S_{n}}(\omega) a^{+}(1, \omega)$ :

$$
\lim _{n \rightarrow \infty} \int_{S_{n}} a^{+}(1, \omega) d \mu=\int_{\Omega} \lim _{n \rightarrow \infty} \mathcal{X}_{S_{n}}(\omega) a^{+}(1, \omega) d \mu=0
$$

contradizendo a hipótese.

Com base em duas afirmações concluimos que existem $\delta>0$ e $N>0$ tais que $\mu\left(B_{n}\right) \geq \delta$ para todo $n>N$, e o lema segue do argumento no início da demonstração.

\subsection{Prova do Lema de Pliss Ergódico}

Prova da Proposição 7.1. Seja $\epsilon>0$, definimos o cociclo:

$$
c(n, \omega):=a(n, \omega)-(A-\epsilon) n .
$$

Notemos que:

1. $c$ é subaditivo. De fato:

$$
\begin{aligned}
c(n+m, \omega) & =a(n+m, \omega)-(A-\epsilon)(n+m) \\
& \leq\left[a\left(n, T^{m} \omega\right)-(A-\epsilon) n\right]+[a(m, \omega)-(A-\epsilon) m] \\
& \leq c\left(n, T^{m} \omega\right)+c(m, \omega) .
\end{aligned}
$$

2. $\tilde{A}:=\lim _{n \rightarrow \infty} \frac{1}{n} \int_{\Omega} c(n, \omega) d \mu=\epsilon>0$. De fato:

$$
\tilde{A}=\lim _{n \rightarrow \infty} \frac{1}{n} \int_{\Omega} a(n, \omega) d \mu-(A-\epsilon)=A-(A-\epsilon)=\epsilon .
$$

3. $c(n, \omega)-c\left(n-k, T^{k} \omega\right) \geq 0 \Longleftrightarrow a(n, \omega)-a\left(n-k, T^{k} \omega\right) \geq(A-\epsilon) k$.

Aplicando o Lema de Pliss Fraco 7.3 ao cociclo $c(n, \omega)$, concluimos que $\mu\left(E_{\epsilon}\right)>0$.

Afirmação 7.6. Sejam $l \in \mathbb{N} e \tilde{\epsilon} \in \mathbb{R} \operatorname{com} 0<\tilde{\epsilon}<\epsilon$. Então $T^{l} E_{\tilde{\epsilon}} \subset E_{\epsilon}$. 
Prova da Afirmação 7.6. Seja $\omega \in E_{\tilde{\epsilon}}$ e tomando a sequência $\left\{\tilde{n}_{i}\right\}_{i \in \mathbb{N}} \rightarrow \infty$ tal que:

$$
a\left(\tilde{n}_{i}, \omega\right)-a\left(\tilde{n}_{i}-\tilde{k}, T^{\tilde{k}} \omega\right) \geq(A-\tilde{\epsilon}) \tilde{k}, \quad \forall \tilde{k} \in\left[\tilde{K}, \tilde{n}_{i}\right]
$$

Seja $n_{i}:=\tilde{n}_{i}-l$. Podemos supor que $n_{i}>0$ descartando alguns elementos da sequência. Tomando $K(\omega, \tilde{\epsilon}):=\max \left\{\tilde{K}(\omega, \epsilon), \frac{(A-\epsilon) l-a(l, \omega)}{\epsilon-\tilde{\epsilon}}\right\}$. Então para todo $k \in\left[K, n_{i}\right]$ temos:

$$
\begin{aligned}
a\left(n_{i}, T^{l} \omega\right)-a\left(n-k, T^{k+l} \omega\right) & \geq a\left(\tilde{n}_{i}, \omega\right)-a\left(\tilde{n}_{i}-(k+l), T^{k+l} \omega\right)-a(l, \omega) \\
& \geq(A-\tilde{\epsilon})(k+l)-a(l, \omega) \\
& \geq(A-\epsilon) k
\end{aligned}
$$

Isto prova que $T^{l} \omega \in E_{\epsilon}$, demonstrando a afirmação.

Definimos o conjunto:

$$
F_{\epsilon}:=\bigcap_{l \geq 0} T^{-l} E_{\epsilon}
$$

Observe que $F_{\epsilon} \subset \bigcap_{l \geq 1} T^{-l} E_{\epsilon}=T^{-1} F_{\epsilon}$, como $T$ preserva a medida, temos que $F_{\epsilon}$ é um conjunto invariante $\bmod 0$, portanto $\mu\left(F_{\epsilon}\right)=1$. Usando a definição de $F_{\epsilon}$ temos $\mu\left(E_{\epsilon}\right)=1$ para todo $\epsilon>0$. Como $E_{\tilde{\epsilon}} \subset E_{\epsilon}$ quando $\tilde{\epsilon} \leq \epsilon$ temos:

$$
E:=\bigcap_{\epsilon>0} E_{\epsilon} \quad \text { tem medida } 1
$$

\subsection{Preparatórios para a demonstração do Teorema de Kingman}

Proposição 7.7. Seja a um cociclo subaditivo. Então para todo $M \in \mathbb{N}^{*}$ e quase todo $\omega \in \Omega$ temos:

$$
\begin{aligned}
\limsup _{n \rightarrow \infty} \frac{1}{n} a(n, \omega) & =\limsup _{n \rightarrow \infty} \frac{1}{n M} a(n M, \omega), \\
\liminf _{n \rightarrow \infty} \frac{1}{n} a(n, \omega) & =\liminf _{n \rightarrow \infty} \frac{1}{n M} a(n M, \omega) .
\end{aligned}
$$

O lema abaixo normalmente é obtido como consequência do Teorema de Birkhoff 1.2. Seguindo [Avila e Bochi, 2009], apresentaremos uma prova do lema que não faz uso deste teorema. O interesse disso é obter uma prova do Teorema de Kingman (assim como a prova do Lema de Pliss ergódico) 
independe do Teorema de Birkhoff. Em particular, reobteremos Birkhoff como corolário.

Lema 7.8. Seja $f: \Omega \rightarrow \mathbb{R}$ uma função integrável. Então:

$$
\lim _{n \rightarrow \infty} \frac{f\left(T^{n} \omega\right)}{n}=0, \quad \text { para quase todo } \omega \in \Omega .
$$

Prova do Lema 7.8. Dado $\epsilon>0$, temos que provar:

$$
\bigcap_{n \in \mathbb{N}} \bigcup_{k \geq n}\left[\left|f \circ T^{k}\right| \geq \epsilon n\right], \quad \text { tem medida nula. }
$$

Usando a invariância da medida em relação a $T$ :

$$
\begin{aligned}
\sum_{n=1}^{\infty} \mu\left[\left|f \circ T^{n}\right| \geq \epsilon n\right] & =\sum_{n=1}^{\infty} \mu[|f| \leq \epsilon n]=\sum_{n=1}^{\infty} \sum_{k=n}^{\infty} \mu\left[k \geq \frac{|f|}{\epsilon}<k+1\right] \\
& =\sum_{k=1}^{\infty} k \mu\left[k \leq \frac{|f|}{\epsilon}<k+1\right] \leq \int_{[|f|>\epsilon]} \frac{|g|}{\epsilon} d \mu<\infty
\end{aligned}
$$

Aplicando o Teorema de Borel-Cantelli, temos:

$$
\mu\left(\bigcap_{n \in \mathbb{N}} \bigcup_{k \geq n}\left[f \circ T^{k} \geq \epsilon n\right]\right)=0
$$

Prova da Proposição 7.7. Inicialmente fixamos $M$. Dado $n \in \mathbb{N}$, pelo algoritmo da divisão Euclidiana:

$n=q_{n} M+r_{n}=\left(q_{n}+1\right) M-s_{n}$, para algum $q_{n}, r_{n}, s_{n} \in \mathbb{N}$ com $0 \leq r_{n}, s_{n}<M$

Pela subaditividade, temos:

$$
a\left(\left(q_{n}+1\right) M, \omega\right)-a\left(s_{n}, T^{n} \omega\right) \leq a(n, \omega) \leq a\left(q_{n} M, \omega\right)+a\left(r_{n}, T^{q_{n} M} \omega\right),
$$

dividindo por $n$ e como $\left(q_{n}+1\right) M \leq n$, a desigualdade à esquerda de (7.11) implica:

$$
\begin{aligned}
\frac{1}{n} a(n, \omega) & \geq \frac{1}{n} a\left(\left(q_{n}+1\right) M, \omega\right)-\frac{1}{n} a\left(s_{n}, T^{n} \omega\right) \\
& \geq \frac{1}{\left(q_{n}+1\right) M} a\left(\left(q_{n}+1\right) M, \omega\right)-\frac{1}{n} a\left(s_{n}, T^{n} \omega\right) .
\end{aligned}
$$


Analogamente, como $q_{n} M \geq n$, pela desigualdade à direita de (7.11):

$$
\begin{aligned}
\frac{1}{n} a(n, \omega) & \leq \frac{1}{n} a\left(q_{n} M, \omega\right)+\frac{1}{n} a\left(r_{n}, T^{q_{n} M} \omega\right) \\
& \leq \frac{1}{q_{n} M} a\left(q_{n} M, \omega\right)+\frac{1}{n} a\left(r_{n}, T^{q_{n} M} \omega\right) .
\end{aligned}
$$

Tomando limite inferior na desigualdade (7.12) acima, levando em conta que $0 \leq s_{n}<M$ para todo $n \in \mathbb{N}$ e aplicando o Lema 7.8 :

$$
\begin{aligned}
\liminf _{n \rightarrow \infty} \frac{1}{n} a(n, \omega) & \geq \liminf _{n \rightarrow \infty} \frac{1}{\left(q_{n}+1\right) M} a\left(\left(q_{n}+1\right) M, \omega\right)-\limsup _{n \rightarrow \infty} \frac{1}{n} a\left(s_{n}, T^{n} \omega\right) \\
& =\liminf _{n \rightarrow \infty} \frac{1}{\left(q_{n}+1\right) M} a\left(\left(q_{n}+1\right) M, \omega\right) \\
& =\liminf _{n \rightarrow \infty} \frac{1}{n M} a(n M, \omega) .
\end{aligned}
$$

Analogamente, tomando limite superior (7.13), e aplicando o Lema 7.8:

$$
\begin{aligned}
\limsup _{n \rightarrow \infty} \frac{1}{n} a(n, \omega) & \leq \limsup _{n \rightarrow \infty} \frac{1}{q_{n} M} a\left(q_{n} M, \omega\right)+\limsup _{n \rightarrow \infty} \frac{1}{n} a\left(r_{n}, T^{q_{n} M} \omega\right) \\
& =\limsup _{n \rightarrow \infty} \frac{1}{q_{n} M} a\left(q_{n} M, \omega\right) \\
& =\limsup _{n \rightarrow \infty} \frac{1}{n M} a(n M, \omega) .
\end{aligned}
$$

Isso conclui a prova da proposição.

O lema a seguir é um corolário do Lema de Pliss Ergódico, note que a segunda parte do Lema nada mais é do que o Teorema de Birkhoff 1.2:

Lema 7.9. Sob as hipóteses da Proposição 7.1, se $A>-\infty$ temos:

$$
\liminf _{n \rightarrow \infty} \frac{a(n, \omega)}{n} \geq A, \quad \text { para quase todo } \omega \in \Omega
$$

Em particular, se $a(n, \omega)$ for aditivo então:

$$
\lim _{n \rightarrow \infty} \frac{a(n, \omega)}{n}=A, \quad \text { para quase todo } \omega \in \Omega
$$

Prova. Dado $\epsilon>0$, aplicando a Proposição 7.1, para quase todo $\omega \in \Omega$ existem $K$ e uma sequência $\left\{n_{i}\right\}_{i \in \mathbb{N}} \rightarrow \infty$ :

$$
a\left(n_{i}, \omega\right)-a\left(n_{i}-k, T^{k} \omega\right) \geq(A-\epsilon) k
$$


para todo $K \leq k \leq n_{i}$. Escolhendo $k=n_{i}$ obtemos:

$$
\begin{aligned}
& a\left(n_{i}, \omega\right) \geq(A-\epsilon) n_{i} \\
\Longrightarrow & \liminf _{n \rightarrow \infty} \frac{a(n, \omega)}{n} \geq(A-\epsilon) .
\end{aligned}
$$

Considerando uma sequência de $\left\{\epsilon_{n}\right\}_{n \in \mathbb{N}}$ tendendo a 0 , concluímos:

$$
\liminf _{n \rightarrow \infty} \frac{a(n, \omega)}{n} \geq A, \quad \text { para quase todo } \omega \in \Omega \text {. }
$$

Caso $a(n, \omega)$ for aditivo, então $-a(n, \omega)$ é subaditivo, pelos mesmos argumentos temos:

$$
\begin{aligned}
& \liminf _{n \rightarrow \infty}\left(-\frac{a(n, \omega)}{n}\right) \geq-A, \quad \text { para quase todo } \omega \in \Omega, \\
\Longrightarrow & \limsup _{n \rightarrow \infty} \frac{a(n, \omega)}{n} \leq A, \quad \text { para quase todo } \omega \in \Omega,
\end{aligned}
$$

com isso mostramos a segunda parte da afirmação.

\subsection{Prova do Teorema de Kingman}

Teorema 7.10 (Kingman). Seja a um cociclo subaditivo. Supondo que T é ergódico e $A>-\infty$. Então existe:

$$
\lim _{n \rightarrow \infty} \frac{1}{n} a(n, \omega)=A, \quad \text { para quase todo } \omega \in \Omega .
$$

Prova. Pelo Lema 7.9, a prova se reduz em mostrar que dado qualquer $\epsilon>0$, temos:

$$
\limsup _{n \rightarrow \infty} \frac{a(n, \omega)}{n}-\liminf _{n \rightarrow \infty} \frac{a(n, \omega)}{n} \leq \epsilon, \quad \text { para quase todo } \omega \in \Omega
$$

Usando a Proposição 2.8, existe $M(\epsilon) \in \mathbb{N}$ tal que:

$$
\frac{1}{M} \int_{\Omega} a(M, \omega) d \mu \leq A+\epsilon
$$

com isso definimos:

$$
a^{M}(n, \omega):=\frac{1}{M}\left(a(n M, \omega)-\sum_{i=0}^{n-1} a\left(M, T^{i M} \omega\right)\right)
$$


Afirmação 7.11. A aplicação $a^{M}(n, \omega)$ é um cociclo subaditivo não-positivo $\left(a^{M} \leq 0\right)$ com respeito à transformação $T^{M}$.

Prova da Afirmação 7.11. A conta abaixo verifica a subaditividade de $a^{M}$ :

$$
\begin{aligned}
M a^{M}(n+m, \omega)= & a((n+m) M, \omega)-\sum_{i=0}^{n+m-1} a\left(M, T^{i M} \omega\right) \\
\leq & \left(a\left(n M, T^{m M} \omega\right)-\sum_{i=0}^{n-1} a\left(M, T^{i M+m M} \omega\right)\right) \\
& +\left(a(m M, \omega)-\sum_{i=0}^{m-1} a\left(M, T^{i M} \omega\right)\right) \\
\leq & M a^{M}\left(n, T^{m M} \omega\right)+M a^{M}(m, \omega) .
\end{aligned}
$$

Usando a subaditividade do cociclo $a$ :

$$
a(n M, \omega) \leq \sum_{i=0}^{n-1} a\left(M, T^{i M} \omega\right)
$$

concluimos que $a^{M}(n, \omega)$ é não-positivo.

Voltando à prova do Teorema, notemos que:

$$
\begin{aligned}
\int_{\Omega} a^{M}(n, \omega) d \mu & =\frac{1}{M} \int_{\Omega} a(n M, \omega) d \mu-\sum_{i=0}^{n-1} \frac{1}{M} \int_{\Omega} a\left(M, T^{i M} \omega\right) d \mu \\
& \geq \frac{a_{n M}}{M}-n(A+\epsilon) .
\end{aligned}
$$

Portanto o drift $\tilde{A}$ do cociclo $a^{M}$ satisfaz a seguinte desigualdade:

$$
\tilde{A}:=\lim _{n \rightarrow \infty} \frac{1}{n} \int_{\Omega} a^{M}(n, \omega) d \mu \geq \lim _{n \rightarrow \infty} \frac{a_{n M}}{n M}-(A+\epsilon)=-\epsilon .
$$

Usando o Lema 7.9 e a desigualdade acima:

$$
\liminf _{n \rightarrow \infty} \frac{1}{n M} a^{M}(n, \omega) \geq \tilde{A}=-\epsilon .
$$

Por outro lado, notemos que $\sum_{i=0}^{n-1} a\left(M, T^{i M} \omega\right)$ é um cociclo aditivo, pelo Lema 7.9 novamente, temos:

$$
\lim _{n \rightarrow \infty} \frac{1}{n M} \sum_{i=0}^{n-1} a\left(M, T^{i M} \omega\right), \quad \text { existe e é número real. }
$$


Agora usando a equação acima, (7.15), a Proposição 7.7 e a propriedade de $a^{M} \leq 0$ :

Agora com a Proposição 7.7 (na primeira linha), a equação acima (na segunda linha), a definição de $a^{M}$ (equação (7.14) na terceira linha), a equação (7.15) e a propriedade de $a^{M} \leq 0$ (na última linha) obtemos:

$$
\begin{aligned}
\limsup _{n \rightarrow \infty} \frac{1}{n} a(n, \omega) & -\liminf _{n \rightarrow \infty} \frac{1}{n} a(n, \omega)=\underbrace{\limsup _{n \rightarrow \infty} \frac{1}{n M} a(n M, \omega)-\liminf _{n \rightarrow \infty} \frac{1}{n M} a(n M, \omega)}_{(\star)} \\
& =(\star)+\lim _{n \rightarrow \infty} \frac{1}{n M} \sum_{i=0}^{n-1} a\left(M, T^{i M} \omega\right)-\lim _{n \rightarrow \infty} \frac{1}{n M} \sum_{i=0}^{n-1} a\left(M, T^{i M} \omega\right) \\
& =\limsup _{n \rightarrow \infty} \frac{1}{n} a^{M}(n, \omega)-\liminf _{n \rightarrow \infty} \frac{1}{n} a^{M}(n, \omega) \\
& \leq-\liminf _{n \rightarrow \infty} \frac{1}{n} a^{M}(n, \omega) \leq \epsilon .
\end{aligned}
$$

Como $\epsilon>0$ é arbitrário, concluímos a demonstração. 


\section{Prova do Teorema}

Neste capítulo provaremos o Teorema de K-M 2.10. Durante a demonstração usaremos somente a noção de curvatura não-positiva (Definição 2.5), o Lema de Pliss Ergódico 7.1, o Teorema de Kingman 7.10 e o Lema Geométrico 6.1 (em cuja prova usamos a convexidade uniforme).

\subsection{Escolhendo $\omega$ com boas propriedades}

Enunciamos novamente a segunda parte do Teorema 2.10:

Afirmação 8.1. Se $A>0$, então para quase todo $\omega \in \Omega$ existe uma única geodésica de velocidade unitária $\gamma(\cdot, \omega)$ em $Y$, tal que $\gamma(0, \omega)=$ y e:

$$
\lim _{n \rightarrow \infty} \frac{d\left(\gamma(n A, \omega), y_{n}(\omega)\right)}{n}=0 .
$$

Preparação. Definimos o cociclo subaditivo $a(n, \omega):=d\left(y, y_{n}\right)$. Escolhemos a sequência $\left\{\epsilon_{i}>0\right\}_{i \in \mathbb{N}}$ satisfazendo as duas condições abaixo:

$$
\begin{aligned}
& h\left(\frac{2 \epsilon_{i}}{A-\epsilon_{i}}\right) \leq 2^{-i}, \quad \forall i \in \mathbb{N} \\
& \lim _{i \rightarrow \infty} \epsilon_{i}=0,
\end{aligned}
$$

onde $h$ é a função do Lema Geométrico 6.1. As escolhas acima são possíveis pois $\lim _{t \rightarrow 0} h(t)=0$ e $A>0$.

Fixamos $\omega \in \Omega$ de modo que valem o Lema de Pliss Ergódico (Proposição 7.1) e o Teorema de Kingman 7.10. Logo para qualquer $\epsilon_{i}>0$, existem sequência crescente $\left\{m_{i, j}\right\}_{j \in \mathbb{N}} \rightarrow \infty$, constantes $L_{i}$ e $\tilde{L}_{i}$ tais que:

$$
\begin{aligned}
& a\left(m_{i, j}, \omega\right)-a\left(m_{i, j}-k, T^{k} \omega\right) \geq\left(A-\epsilon_{i}\right) k, \quad \forall k, L_{i} \leq k \leq m_{i, j}, \\
& \left(A-\epsilon_{i}\right) k \leq a(k, \omega) \leq\left(A+\epsilon_{i}\right) k, \quad \forall k, \tilde{L}_{i} \leq k,
\end{aligned}
$$




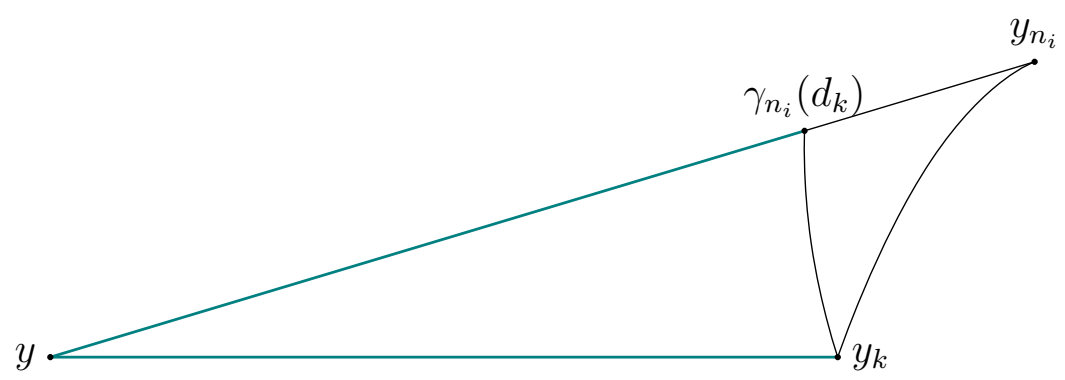

Figura 8.1: Um esboço dos pontos, aplicaremos o Lema 6.1 neste triângulo.

Definimos $K_{i}:=\max \left\{L_{i}, \tilde{L}_{i}\right\}$, e definimos a sequência $\left\{n_{i}\right\}_{i \in \mathbb{N}}$ colocando:

$$
\begin{aligned}
& n_{0}:=\min \left\{m_{0, j} \mid \text { tal que } m_{0, j}>\max \left\{K_{0}, K_{1}\right\}, j \in \mathbb{N}\right\}, \\
& n_{i}:=\min \left\{m_{i, j} \mid \text { tal que } m_{i, j}>\max \left\{K_{i}, K_{i+1}, n_{i-1}\right\}, j \in \mathbb{N}\right\}, \quad \forall i \in \mathbb{N}^{*} .
\end{aligned}
$$

Note que as definições acima garantem:

$$
\bigcup_{i \in \mathbb{N}}\left[K_{i}, n_{i}\right]=\left[K_{0}, \infty\right)
$$

Com as escolhas e propriedades acima, estamos aptos a mostrar a existência da geodésica $\gamma$.

\subsection{Construção da Sequência de Cauchy}

Afirmação 8.2. Para cada $n \in \mathbb{N}$, seja $\gamma_{n}$ a geodésica com velocidade unitário começando de y e passando por $y_{n}$. Então para cada $t \in \mathbb{R}^{+}$fixo, a sequência $\left\{\gamma_{n_{i}}(t)\right\}_{i \in \mathbb{N}}$ é de Cauchy.

Prova. Somando a desigualdade (8.2) a desigualdade à direita de (8.3), obtemos:

$$
\begin{aligned}
& a(k, \omega)+\left(A-\epsilon_{i}\right) k \leq\left(A+\epsilon_{i}\right) k+a\left(n_{i}, \omega\right)-a\left(n_{i}-k, T^{k} \omega\right) \\
\Longrightarrow & a(k, \omega)+a\left(n_{i}-k, T^{k} \omega\right) \leq a\left(n_{i}, \omega\right)+2 \epsilon k .
\end{aligned}
$$

A propriedade de semi-contração implica que $d\left(y_{k}, y_{n_{i}}\right) \leq a\left(n_{i}-k, T^{k} \omega\right)$. A partir da definição de $a$, e da desigualdade à esquerda de (8.3), a desigualdade acima fica:

$$
\begin{aligned}
d\left(y, y_{k}\right)+d\left(y_{k}, y_{n_{i}}\right) & \leq d\left(y, y_{n_{i}}\right)+2 \epsilon_{i} k \\
& \leq d\left(y, y_{n_{i}}\right)+\frac{2 \epsilon_{i}}{A-\epsilon_{i}} d\left(y, y_{k}\right)
\end{aligned}
$$

Daqui em diante, passaremos a usar a notação $d_{k}:=d\left(y, y_{k}\right)$. 
Pelo Lema Geométrico 6.1 (cf. Fig. 8.1) para $\delta_{i}:=\frac{2 \epsilon_{i}}{A-\epsilon_{i}}$, e a equação (8.1) concluimos que:

$$
d\left(\gamma_{n_{i}}\left(d_{k}\right), y_{k}\right) \leq h\left(\delta_{i}\right) d_{k} \leq 2^{-i} d_{k} \quad \text { para todo } k, \text { com } K_{i} \leq k \leq n_{i} .
$$

Tomemos $i$ suficientemente grande de modo que $d_{n_{i}}>t$. Tomando $k=n_{i}$ e trocando $i$ por $i+1$ na desigualdade acima, temos:

$$
d\left(\gamma_{n_{i+1}}\left(d_{n_{i}}\right), \gamma_{n_{i}}\left(d_{n_{i}}\right)\right)=d\left(\gamma_{n_{i+1}}\left(d_{n_{i}}\right), y_{n_{i}}\right) \leq 2^{-(i+1)} d_{n_{i}}
$$

Como $Y$ possui curvatura não-positiva, vale a desigualdade (2.4), junto com a desigualdade acima, obtemos:

$$
d\left(\gamma_{n_{i+1}}(t), \gamma_{n_{i}}(t)\right) \leq 2^{-(i+1)} t
$$

Pela desigualdade triangular e indução temos:

$$
d\left(\gamma_{n_{i+m}}(t), \gamma_{n_{i}}(t)\right) \leq \sum_{j=1}^{m} 2^{-(i+j)} t \leq 2^{-i} t \quad \forall m \in \mathbb{N}^{*}
$$

Portanto $\left\{\gamma_{n_{i}}(t)\right\}_{i \in \mathbb{N}}$ é uma sequência de Cauchy para cada $t \in \mathbb{R}^{+}$fixo.

Como $Y$ é espaço métrico completo, a sequência $\left\{\gamma_{n_{j}}(t)\right\}_{j \in \mathbb{N}}$ com $t \in$ $[0, \infty)$ converge a uma curva $\gamma:[0, \infty) \rightarrow Y$.

\subsection{Existência da geodésica $\gamma$ com as propriedades do teorema}

Afirmação 8.3. $\gamma$ é uma geodésica de velocidade unitária.

Prova da Afirmação 8.3. De fato, dados $s, t \in \mathbb{R}$ e $\epsilon>0$ existe $n_{i}$ tais que:

$$
\max \left\{d\left(\gamma(s), \gamma_{n_{i}}(s)\right), d\left(\gamma(t), \gamma_{n_{i}}(t)\right)\right\} \leq \frac{\epsilon}{2}
$$

Portanto:

$$
\begin{aligned}
d(\gamma(s), \gamma(t)) & \leq d\left(\gamma(s), \gamma_{n_{i}}(s)\right)+d\left(\gamma_{n_{i}}(s), \gamma_{n_{i}}(t)\right)+d\left(\gamma_{n_{i}}(s), \gamma(t)\right) \\
& \leq \frac{\epsilon}{2}+|s-t|+\frac{\epsilon}{2}=|s-t|+\epsilon
\end{aligned}
$$

Como $\epsilon, s, t$ são arbitrários, temos $|\gamma(s)-\gamma(t)|=|s-t|$ para todos $s, t \in$ $\mathbb{R}^{+}$.

Afirmação 8.4. $\lim _{k \rightarrow \infty} \frac{d\left(\gamma(A k), y_{k}\right)}{k}=0$. 


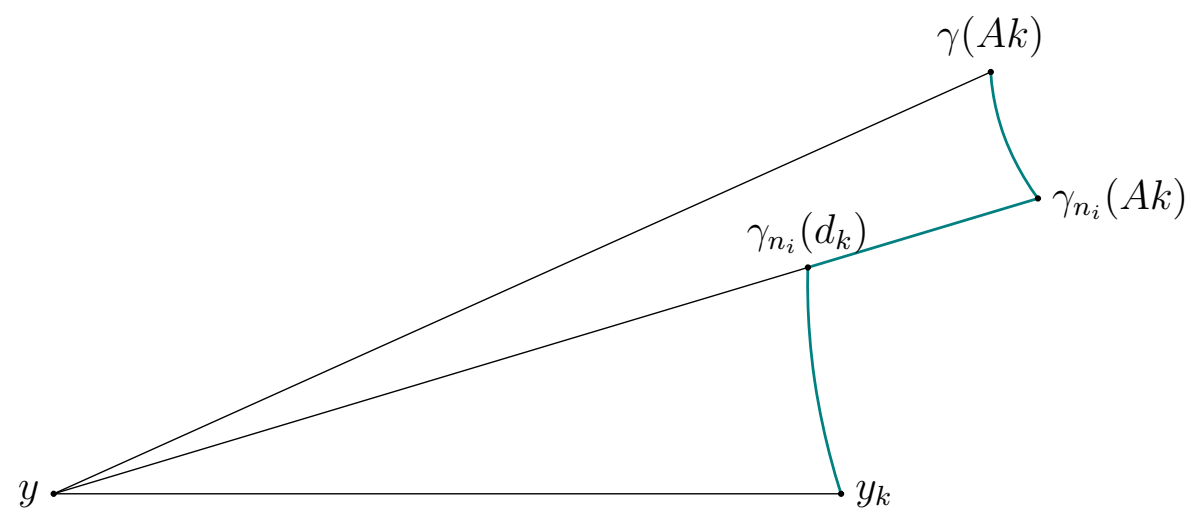

Figura 8.2: Um esboço dos pontos e as geodésicas usadas na demonstração.

Prova da Afirmação 8.4. Dado $k \in \mathbb{N}$, com $k \geq K_{0}$, pela propriedade (8.4) existe $i \in \mathbb{N}$ tal que $K_{i} \leq k \leq n_{i}$. Abaixo usamos a desigualdade triangular na $1^{\underline{a}}$ desigualdade, (8.5) e (8.6) na $2^{\underline{a}}$ desigualdade, (8.3) na $3^{\underline{a}}$ desigualdade obtendo:

$$
\begin{aligned}
d\left(\gamma\left(A k, y_{k}\right)\right) & \leq d\left(\gamma(A k), \gamma_{n_{i}}(A k)\right)+d\left(\gamma_{n_{i}}(A k), \gamma_{n_{i}}\left(d_{k}\right)\right)+d\left(\gamma_{n_{i}}\left(d_{k}\right), y_{k}\right) \\
& \leq 2^{-i} A k+\left|A k-d_{k}\right|+2^{-i} d_{k} \\
& \leq 2^{-i} A k+\epsilon_{i} k+2^{-i}\left(A+\epsilon_{i}\right) k \\
& \leq\left(2^{-i+1} A+2 \epsilon_{i}\right) k .
\end{aligned}
$$

Os termos da conta acima estão desenhados na Figura 8.2. Portanto concluímos que:

$$
\limsup _{k \rightarrow \infty} \frac{d\left(\gamma(A k), y_{k}\right)}{k} \leq \limsup _{i \rightarrow \infty}\left(2^{-i+1} A+2 \epsilon_{i}\right)=0 .
$$

A unicidade da geodésica $\gamma$ segue de (2.4). Assim completamos a prova da segunda parte do Teorema 2.10. 


\section{Referências Bibliográficas}

[Avila e Bochi, 2009] AVILA, A.; BOCHI, J.. On the subadditive ergodic theorem. Preprint, 2009.

[Birkhoff, 1931] BIRKHOFF, G. D.. Proof of the ergodic theorem. Proc Natl Acad Sci USA, 17:656-660, 1931.

[Bollobás] BOLLOBÁS, B.. Modern Graph Theory. Springer-Verlag, 1998. [Furstenberg, 1963] FURSTENBERG, H.. Non-commuting random products. Trans. AMS, 108:377-428, 1963.

[Jost] JOST, J.. Nonpositive Curvature: Geometric and Analytic Aspects. Birkhäuser, 1997.

[Kaimanovich, 1989] KAIMANOVICH, V. A.. Lyapunov expoents, symmetric spaces and a multiplicative ergodic theorem for semisimple Lie groups. J. Soviet Math, 47:2387-2398, 1989.

[Karlsson e Margulis, 1999] KARLSSON, A.; MARGULIS, G. A.. A multiplicative ergodic theorem and nonpositively curved spaces. Communications in Mathematical Physics, 208:107-123, 1999.

[Karlsson e Ledrappier, 2008] KARLSSON, A.; LEDRAPPIER, F.. Noncommutative ergodic theorems. Chicago Lectures in Math., p. 396418, 2011.

[Kingman, 1968] KINGMAN, J. F. C.. The ergodic theory of subadditive stochastic process. Royal Statistical Society, 30:499-510, 1968.

[Lang] LANG, S.. Fundamentals of Differential Geometry. SpringerVerlag, 1999.

[M. R. Bridson e A. Haefliger] BRIDSON, M. R.; HAEFLIGER, A.. Metric Spaces of Non-Positive Curvature. Springer-Verlag, 1999.

[Mañé] MAÑÉ, R.. Teoria Ergódica. IMPA, 1983.

[Navas, 2011] NAVAS, A.. An $L^{1}$ ergodic theorem with values in a nonpositively curved space via a canonical barycenter map. a aparecer in Erg. Theory and Dyn. Systems, arXiv:1104.5188, 2011.

[Needham] NEEDHAM, T.. Visual Complex Analysis. Oxford University Press, 1999. 170. 
[Oseledets, 1968] OSELEDETS, V. I.. A multiplicative ergodic theorem. Lyapunov characteristic numbers for dynamical systems. Trudy Moskov, 19:179-210, 1968.

[Tiozzo, 2012] TIOZZO, G.. Sublinear deviation between geodesics and sample paths. Preprint, arXiv:1210.7352, 2012.

[do Carmo] DO CARMO, M. P.. Riemannian Geometry. Birkhäuser, 1992.

[von Neumann, 1932] VON NEUMANN, J.. Proof of the quasi-ergodic hypothesis. Proc Natl Acad Sci USA, 18:70-82, 1932. 